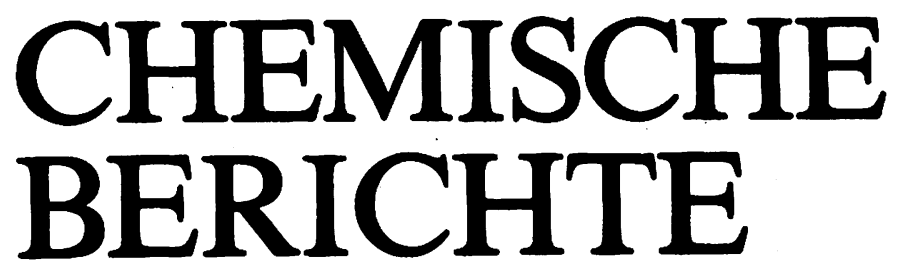

GEGRÜNDET 1868

119. JAHRGANG

HERAUSGEGEBEN IM AUFTRAG DER

GESELLSCHAFT DEUTSCHER CHEMIKER

VON

K. HAFNER - W. KIRMSE - H. MUSSO - H. NÖTH -

J. SAUER - E. WINTERFELDT

UNTER MITWIRKUNG VON

W. BECK - H. A. BRUNE - H. BUDZIKIEWICZ - W. LƯTTKE

REDAKTION: R. TEMME

mit H. SCHILL, J. STREHLOW und A. WIELAND

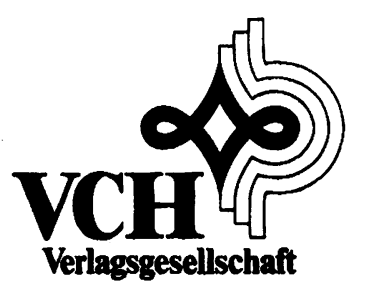




\section{BEMERKUNGEN DER REDAKTION}

1. Die Chemischen Berichte (zu zitieren als Chem. Ber.) setzen die Berichte der Deutschen Chemischen Gesellschaft (zu zitieren als Ber. Dtsch. Chem. Ges.) fort.

2. Die „Berichte“ enthalten Originalmitteilungen aus allen Gebieten der Chemie.

3. Die „Berichte“ erscheinen monatlich; ein Registerheft beschließt jeden Jahrgang.

4. Die Verantwortung für ihre Mitteilungen tragen die Verfasser selbst. - Der Korrespondenzautor ist durch einen hochgestellten Stern hervorgehoben. Die Zugehörigkeit der Autoren zu den Instituten ist durch hochgestellte Kleinbuchstaben markiert.

5. Es werden grundsätzlich nur Arbeiten aufgenommen, die vorher weder im Inland noch im Ausland veröffentlicht worden sind.

6. Eine Anweisung zur Abfassung von Manuskripten für die „Berichte“ wird auf Wunsch zugestellt.

7. Manuskripte sind zu senden an Redaktion der Chemischen Berichte. Dr. R. Temme, Am Klopferspitz, D-8033 Martinsried, Telefon (089) 85783830.

8. Der Eingang der Abhandlungen wird den Autoren am Tage der Registrierung angezeigt.

9. Es werden nur Manuskripte in deutscher oder englischer Sprache aufgenommen. Allen Beiträgen ist eine knappe Zusammenfassung (summary) in beiden Sprachen voranzustellen, einschließlich Titel in der jeweils zweiten Sprache.

10. Der Autor muß das alleinige Urheberrecht besitzen. Mit der Annahme des Manuskriptes durch die Redaktion überträgt er der VCH Verlagsgesellschaft das ausschließliche Nutzungsrecht, insbesondere das Recht der Vervielfältigung wie Fotokopie, Mikrofilm oder mit irgendeinem anderen Verfahren - oder das Manuskript in eine von Maschinen, insbesondere von Datenverarbeitungsmaschinen, verwendbare Sprache zu übergeben oder zu übersetzen (auch in fremde Sprachen).

11: Den Autoren werden 75 Sonderdrucke unentgeltlich portofrei zugesandt. Wünscht ein Autor mehr als 75 Abzüge, so ist dies auf dem Manuskript oder spätestens bei Rücksendung der Korrektur auf dieser zu vermerken. Den Autoren werden nur die Selbstkosten für die Zahl der die Freiexemplare überschreitenden Sonderabzüge berechnet.

12. Anfragen nach dem Verbleib nicht eingetroffener Berichte-Hefte oder Sonderdrucke sind zu richten an: VCH Verlagsgesellschaft mbH, Pappelallee 3, Postfach 1260/1280, D-6940 Weinheim, Telefon (06201) 602-0.

\footnotetext{
Die Chemischen Berichte erscheinen monatlich. Der Jahresbezugspreis beträgt 940. - DM, Einzelheft 105. - DM zuzüglich Versandkosten. In diesen Preisen ist die Mehrwertsteuer enthalten. Die Bezugsbedingungen für Mitglieder der Gesellschaft Deutscher Chemiker werden auf Anfrage von der Geschäftsstelle, Carl-Bosch-Haus, Varrentrappstraße 40-42, Postfach 900440, D-6000 Frankfurt 90, mitgeteilt. Bestellungen richten Sie bitte an Ihre Fachbuchhandlung oder unmittelbar an den Verlag. Abbestellungen nur bis spätestens 3 Monate vor Ablauf des Kalenderjahres. Die Lieferung erfolgt auf Rechnung und Gefahr des Empfängers. Gerichtsstand und Erfüllungsort: Weinheim.

Verlag und Anzeigenabteilung: VCH Verlagsgesellschaft mbH, Postfach 1260/1280, D-6940 Weinheim.

Telefon (06201) 602-0, Telex 465516 vchwh d, Telefax (06201) 602328.

For the USA and Canada: Published monthly by VCH Verlagsgesellschaft mbH, Weinheim, Federal Republic of Germany. Airfreighted and mailed by Publications Expediting Inc., 200 Meacham Avenue, Elmont NY 11003 . Second-class postage paid at Jamaica NY 11431. Annual subscription price: US \$ 478.00 including postage and handling charges. Reduced rate for individual members of the American Chemical Society on request. Subscribers should place their orders through VCH Publishers, Inc., 303 N.W. 12th Avenue, Deerfield Beach FL 33442-1705; Telex 5101011104 VCHPIJB; Phone (305) 428-5566. - Printed in the Federal Republic of Germany.
}

U. S. Postmaster: Send address changes to VCH Publishers, Inc., 303 N.W. 12th Avenue, Deerfield Beach FL 33442-1705. 


\section{INHALT VON HEFT 8}

119. Jahrgang 1986

\section{ANORGANISCHE CHEMIE}

Schwab Michael und Sundermeyer Wolfgang: Bis(trifluormethyl)sulfin, $\left(\mathrm{CF}_{3}\right)_{2} \mathrm{C}=\mathrm{SO}$ : Additionsreaktionen mit Aminen, Alkoholen, Halogenwasserstoffen und

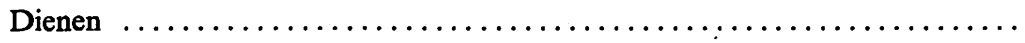

Appel Rolf, Winkhaus Volker und Knoch Falk: Niederkoordinierte Phosphor-Verbindungen, 48: Synthese von 1-Phosphaallenen ................ 2466

Baudler Marianne, Becher Rainer und Germeshausen Joachim: Beiträge zur Chemie des Phosphors, 166: $\mathrm{NaP}_{21} \mathbf{R}_{2}\left(\mathrm{R}=\mathrm{Me}, \mathrm{Et}\right.$, iPr) und $\mathrm{NaP}_{21}\left(\mathrm{SiMe}_{3}\right)_{2}-$ die ersten teilmetallierten Henicosaphosphane(3) ...................

Nöth Heinrich, Otto Peter und Storch Wolfgang: Beiträge zur Chemie des Bors, 176: Zur Synthese Bor-funktioneller Diborylamine über (Stannylamino)borane ....

Lehmkuhl Herbert, Danowski Friedhelm, Benn Reinhard, Mynott Richard und Schroth Gerhard: ( $\eta^{5}$-Cyclopentadienyl)methylnickel-Komplexe mit Ein- und Mehrfach-Doppelbindungssystemen $\ldots \ldots \ldots \ldots \ldots \ldots \ldots \ldots \ldots \ldots$

Roland Eckehart, Bernhardt Wolfgang und Vahrenkamp Heinrich: Überdachungsreaktionen von $\mathrm{RuCo}_{2}(\mathrm{CO})_{11}$ mit Haupt- und Nebengruppenelement-Reagenzien

Conradi Elke, Bohrer Rainer und Müller Ulrich: Direkte Bildung von 2,5-Dimethyl3,3a,4-trithia-1-azapentalenium- und 4,6-Dimethyl-3-thioxo-3H-1,2-dithiolo[4,3-c]pyridinium-Ionen aus Acetonitril und Schwefelwasserstoff. Die Kristallstrukturen ihrer Salze mit $\left[\mathrm{UBr}_{6}\right]^{2 \ominus}$ bzw. $\left[\mathrm{UO}_{2} \mathrm{Br}_{4}\right]^{2 \ominus}$

Appel Rolf, Casser Carl und Knoch Falk: Niederkoordinierte Phosphorverbindungen, 46: 2,4-Diphospha-1-butene $\ldots \ldots \ldots \ldots \ldots \ldots \ldots \ldots \ldots \ldots \ldots \ldots \ldots \ldots$

Lindner Ekkehard, Mayer Hermann August und Wegner Peter: Neuartige basische Liganden für die homogenkatalytische Methanolcarbonylierung, VI: Die Komplexchemie mehrzähniger (Tetrahydrofurfuryl)phosphane und ihr Einfluß auf die Selektivität und den Umsatz bei der Ethanolsynthese aus Methanol ........

Schumann Herbert, Janiak Christoph, Hahn Ekkehardt, Kolax Christine, Loebel Jörg, Rausch Marvin D., Zuckerman Jerold J. und Heeg Mary Jane: Decabenzylgermanocen, -stannocen und -plumbocen. Synthese und Struktur von luftstabilen Metallocenen der 4. Hauptgruppe des Periodensystems der Elemente ...

Kölle Peter, Nöth Heinrich und Paine Robert T.: Beiträge zur Chemie des Bors, 170: Synthese und Struktur eines 1,3,2,4-Diphosphadiboretans $\ldots \ldots \ldots \ldots \ldots \ldots$

\section{ORGANISCHE CHEMIE}

Habernegg Renate und Severin Theodor: Synthese und Reaktion von $\alpha$-(Dimethyl-

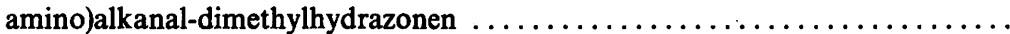

Quast Helmut und Fuchsbauer Hans-Lothar: Intramolekulare Wechselwirkungen in Radikalkationen von Di- und Tetra( $\alpha$-methoxy)-9,10-dihydro-9,10-ethanoan-

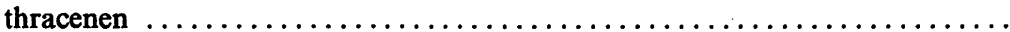


Diehl Klaus, Himbert Gerhard und Henn Lothar: Cycloadditionen, 7: Intramolekulare Diels-Alder-Reaktionen bei Allencarboxaniliden; Variation der Substituenten in $p$-Position des Anilinkerns $\ldots \ldots \ldots \ldots \ldots \ldots \ldots \ldots \ldots \ldots \ldots \ldots \ldots$

Allmann Rudolf, Krestel Magda, Kupfer Rainer und Würthwein Ernst-Ulrich: Synthesen, Struktur und Eigenschaften von $N$-(Alkoxymethylen)carbamidsäu-

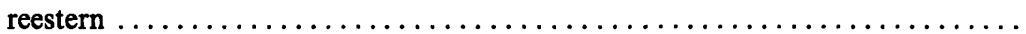

Schwab Michael und Sundermeyer Wolfgang: Bis(trifluormethyl)sulfin, $\left(\mathrm{CF}_{3}\right)_{2} \mathrm{C}=\mathrm{SO}$ : Additionsreaktionen mit Aminen, Alkoholen, Halogenwasserstoffen und

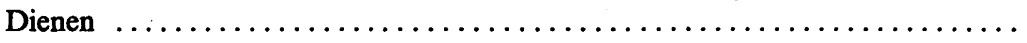

Mayr Herbert und Pock Rudolf: Relative Reaktivität Alkyl-substituierter Alkene und Cycloalkene gegenüber Diarylcarbenium-Ionen $\ldots \ldots \ldots \ldots \ldots \ldots \ldots \ldots$

Pock Rudolf und Mayr Herbert: Relative Reaktivität konjugierter Alkene gegenüber Diarylcarbenium-Ionen $\ldots \ldots \ldots \ldots \ldots \ldots \ldots \ldots \ldots \ldots \ldots \ldots \ldots \ldots \ldots$

Sustmann Reiner und Lau Jürgen: Reduktive Eliminierung aus (2,2'-Bipyridin)diethylpalladium(II), eine mechanistische Studie $\ldots \ldots \ldots \ldots \ldots \ldots \ldots$

Lehmkuhl Herbert, Danowski Friedhelm, Benn Reinhard, Mynott Richard und Schroth Gerhard: ( $\eta^{5}$-Cyclopentadienyl)methylnickel-Komplexe mit Ein- und Mehrfach-Doppelbindungssystemen $\ldots \ldots \ldots \ldots \ldots \ldots \ldots \ldots \ldots \ldots \ldots$

Lösel Walter und Pook Karl-Heinz: 3,4-Dihydroisochinoline, II: 4,5,7,8Tetrahydrocyclopenta[4,5]pyrimido[6,1-a] isochinolin: ein neues Ringsystem durch Umwandlung von 1-(3-Furyl)-3,4-dihydroisochinolin . . . . . . . . .

Fischer Klaus und Hünig Siegfried: Trimethylsiiylcyanid als Umpolungsreagens, XII: Intramolekulare Diels-Alder-Reaktionen über umgepolte Dienale .........

Daub Jörg, Gierisch Sebastian, Klement Ulrich, Knöchel Thomas, Maas Gerhard und Seitz Ulrich: Lichtinduzierte reversible Reaktionen: Synthesen und Eigenschaften photochromer 1,1-Dicyan-1,8a-dihydroazulene und thermochromer 8-(2,2Dicyanvinyl)heptafulvene $\ldots \ldots \ldots \ldots \ldots \ldots \ldots \ldots \ldots \ldots \ldots \ldots \ldots \ldots \ldots \ldots \ldots$

Schill Gottfried, Beckmann Wolfgang, Schweickert Norbert und Fritz Hans: Untersuchungen zur statistischen Synthese von Rotaxanen $\ldots \ldots \ldots \ldots \ldots \ldots$

Wiehl Wolfgang und Frahm August W.: Asymmetrische reduktive Aminierung von Cycloalkanonen, 5: Synthese und absolute Konfiguration 2-substituierter $\mathrm{Cy}$ -

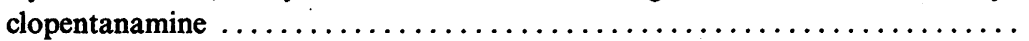

Onistschenko Andreas, Buchholz Berthold und Stamm Helmut: Reaktionen mit Aziridinen, 38: Zur Regioselektivität der nucleophilen Ringöffnung von aktivierten 2-Phenylaziridinen 


\section{INORGANIC CHEMISTRY}

Schwab Michael and Sundermeyer Wolfgang: Bis(trifluoromethyl)sulfine, $\left(\mathrm{CF}_{3}\right)_{2} \mathrm{C}=\mathrm{SO}$ : Addition Reactions with Amines, Alcohols, Hydrogen Halides,

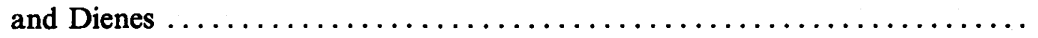

Appel Rolf, Winkhaus Volker, and Knoch Falk: Low Coordinated Phosphorus Compounds, 48: Synthesis of 1-Phosphaallenes $\ldots \ldots \ldots \ldots \ldots \ldots \ldots \ldots \ldots$

Baudler Marianne, Becher Rainer, and Germeshausen Joachim: Contributions to the Chemistry of Phosphorus, 166: $\mathrm{NaP}_{21} \mathbf{R}_{2}(\mathrm{R}=\mathrm{Me}, \mathrm{Et}, \mathrm{iPr})$ and $\mathrm{NaP}_{21}-$ $\left(\mathrm{SiMe}_{3}\right)_{2}$ - The First Partially Metalated Henicosaphosphanes(3) .........

Nöth Heinrich, Otto Peter, and Storch Wolfgang: Contributions to the Chemistry of Boron, 176: The Synthesis of Boron Functional Diborylamines via (Stannyl-

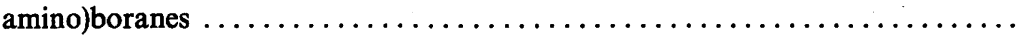

Lehmkuhl Herbert, Danowski Friedhelm, Benn Reinhard, Mynott Richard, and Schroth Gerhard: ( $\eta^{5}$-Cyclopentadienyl)methylnickel Complexes with Single and

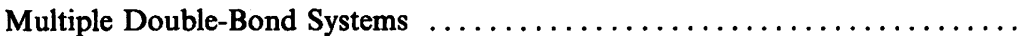

Roland Eckehart, Bernhardt Wolfgang, and Vahrenkamp Heinrich: Capping Reactions of $\mathrm{RuCo}_{2}(\mathrm{CO})_{11}$ with Main Group and Transition Element Reagents ...

Conradi Elke, Bohrer Rainer, and Müller Ulrich: Direct Formation of 2,5-Dimethyl3,3a,4-trithia-1-azapentalenium and 4,6-Dimethyl-3-thioxo-3H-1,2-dithiolo[4,3-c]pyridinium Ions from Acetonitrile and Hydrogen Sulfide. Crystal Structures of their Compounds with $\left[\mathrm{UBr}_{6}\right]^{2 \ominus}$ and $\left[\mathrm{UO}_{2} \mathrm{Br}_{4}\right]^{2 \ominus} \ldots \ldots \ldots \ldots \ldots$

Appel Rolf, Casser Carl, and Knoch Falk: Low Coordinated Phosphorus Compounds,



Lindner Ekkehard, Mayer Hermann August, and Wegner Peter: Novel Basic Ligands for the Homogeneous Catalytic Methanol Carbonylation, VI: The Complex Chemistry of Multidentate (Tetrahydrofurfuryl)phosphanes and their Influence on the Selectivity and Conversion for the Synthesis of Ethanol from Methanol

Schumann Herbert, Janiak Christoph, Hahn Ekkehardt, Kolax Christine, Loebel Jörg, Rausch Marvin D., Zuckerman Jerold J., and Heeg Mary Jane: Decabenzylgermanocene, -stannocene, and -plumbocene. Synthesis and Structure of Air-stable

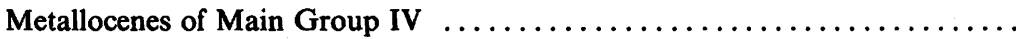

Kölle Peter, Nöth Heinrich, and Paine Robert T.: Contributions to the Chemistry of Boron, 170: Synthesis and Structure of a 1,3,2,4-Diphosphadiboretane ......

\section{ORGANIC CHEMISTRY}

Habernegg Renate and Severin Theodor: Synthesis and Reaction of $\alpha$-(Dimethylamino)alkanal Dimethylhydrazones $\ldots \ldots \ldots \ldots \ldots \ldots \ldots \ldots \ldots \ldots$

Quast Helmut and Fuchsbauer Hans-Lothar: Intramolecular Interactions in Radical Cations of Di- and Tetra( $\alpha$-methoxy)-9,10-dihydro-9,10-ethanoanthracenes ...

Diehl Klaus, Himbert Gerhard, and Henn Lothar: Cycloadditions, 7: Intramolecular Diels-Alder Reactions of Allenecarboxanilides; Variation of the Substituents in the $p$-Position of the Aniline Nucleus $\ldots \ldots \ldots \ldots \ldots \ldots \ldots \ldots \ldots . . \ldots \ldots$ 
Allmann Rudolf, Krestel Magda, Kupfer Rainer, and Würthwein Ernst-Ulrich: Syntheses, Structures, and Properties of $N$-(Alkoxymethylene)carbamates .....

Schwab Michael and Sundermeyer Wolfgang: Bis(trifluoromethyl)sulfine, $\left(\mathrm{CF}_{3}\right)_{2} \mathrm{C}=\mathrm{SO}$ : Addition Reactions with Amines, Alcohols, Hydrogen Halides,

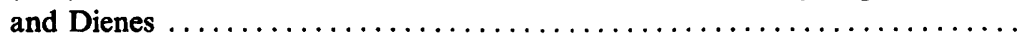

Mayr Herbert and Pock Rudolf: Relative Reactivities of Alkyl-Substituted Alkenes and Cycloalkenes towards Diarylcarbenium Ions $\ldots \ldots \ldots \ldots \ldots \ldots \ldots$

Pock Rudolf and Mayr Herbert: Relative Reactivity of Conjugated Alkenes towards

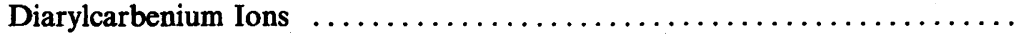

Sustmann Reiner and Lau Jürgen: Reductive Elimination from (2,2'-

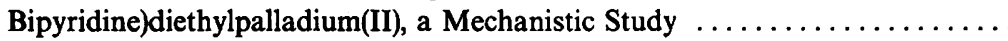

Lehmkuhl Herbert, Danowski Friedhelm, Benn Reinhard, Mynott Richard, and Schroth Gerhard: $\left(\eta^{5}\right.$-Cyclopentadienyl)methylnickel Complexes with Single and Multiple Double-Bond Systems

Lösel Walter and Pook Karl-Heinz: 3,4-Dihydroisoquinolines, II: 4,5,7,8Tetrahydrocyclopenta[4,5]pyrimido[6,1-a]isoquinoline: A Novel Ring System via 1-(3-Furyl)-3,4-dihydroisoquinoline Transformation $\ldots \ldots \ldots \ldots \ldots \ldots$

Fischer Klaus and Hünig Siegfried: Trimethylsilyl Cyanide - A Reagent for Umpolung, XII: Intramolecular Diels-Alder Reaction of Dienals via Umpolung ..

Daub Jörg, Gierisch Sebastian, Klement Ulrich, Knöchel Thomas, Maas Gerhard, and Seitz Ulrich: Light-Induced and Reversible Transformations: Syntheses and Properties of Photochromic 1,1-Dicyano-1,8a-dihydroazulenes and Thermochromic 8-(2,2-Dicyanovinyl)heptafulvenes.$\ldots \ldots \ldots \ldots \ldots \ldots \ldots \ldots \ldots$

Schill Gottfried, Beckmann Wolfgang, Schweickert Norbert, and Fritz Hans: Studies on the Statistical Synthesis of Rotaxanes $\ldots \ldots \ldots \ldots \ldots \ldots \ldots \ldots \ldots$

Wiehl Wolfgang and Frahm August W.: Asymmetric Reductive Amination of Cycloalkanones, 5: Synthesis and Absolute Configuration of 2-Substituted Cyclopentanamines

Onistschenko Andreas, Buchholz Berthold, and Stamm Helmut: Reactions with Aziridines, 38: On the Regioselectivity of the Nucleophilic Ring Opening of Activated 2-Phenylaziridines 


\section{AUTORENREGISTER}

Allmann, R. ............... 2444

Appel, $R$.

2466,2609

Baudler, M. . . . . . . . . 2510

Becher, $R . \ldots \ldots \ldots \ldots \ldots \ldots \ldots 2510$

Beckmann, W. . . . . . . . . . 2647

Benn, R. ................ 2542

Bernhardt, $W . \ldots \ldots \ldots \ldots \ldots \ldots 2566$

Bohrer, $R . \ldots \ldots \ldots \ldots \ldots \ldots .2582$

Buchholz, B. ............... 2678

Casser, C. ................ 2609

Conradi, E. .............. 2582

Danowski, F. . . . . . . . . . . . . 2542

Daub, J. ................. 2631

Diehl, K. ............... 2430

Fischer, $K . \ldots \ldots \ldots \ldots \ldots \ldots \ldots 2590$

Frahm, A. W. ............ 2668

Fritz, $H$. 2647

Fuchsbauer, H.-L. ........... 2414

Germeshausen, J. ........... 2510

Gierisch, $S . \ldots \ldots \ldots \ldots \ldots \ldots \ldots 2631$

Habernegg, R. . . . . . . . . . . . 2397

Hahn, E. 2656

Heeg, M. J. . . ............ 2656

Henn, L. . . . . . . . . . . . . . . . 2430

Himbert, G. . . . . . . . . . . . 2430

Hünig, S. . . . . . . . . . . 2590

Janiak, C. . . . . . . . . . . . . . 2656

Klement, $U . \ldots \ldots \ldots \ldots \ldots \ldots . \ldots 2631$

Knoch, F. . . . . . . . . . 2466, 2609

Knöchel, T. ............. 2631

Kölle, P. . . . . . . . . . . . . . 2681

Kolax, C. ................ 2656

Krestel, M. ................ 2444

Kupfer, R. .............. 2444

Lau, J. .................... 2531
Lehmkuhl, H. . . . . . . . . . . . 2542

Lindner, E. . . . . . . . . . . . 2616

Loebel, J. . . . . . . . . . . . . . . . 2656

Lösel, $W . \ldots \ldots \ldots \ldots \ldots \ldots \ldots . \ldots 2553$

Maas, G. . ............... 2631

Mayer, H. A. ............... 2616

Mayr, H. ............ 2473, 2497

Müller, $U . \ldots \ldots \ldots \ldots \ldots \ldots \ldots 2582$

Mynott, R. . . . . . . . . . . . 2542

Nöth, H. . .......... 2517, 2681

Onistschenko, A. . ........... 2678

Otto, P. . . . . . ........... 2517

Paine, R. T. ................ 2681

Pock, R. ............... 2473, 2497

Pook, K. H. . ............ 2553

Quast, H. . . . . . . . . ........ 2414

Rausch, M. D. . . . . . . . . . . 2656

Roland, E. ............... 2566

Schill, G. .................. 2647

Schroth, G. . . . . . . . . . . . 2542

Schumann, $H . \ldots \ldots \ldots \ldots \ldots \ldots 2656$

Schwab, M. ............. 2458

Schweickert, N. . . . . . . . . . . . 2647

Seitz, $U . \ldots \ldots \ldots \ldots \ldots \ldots \ldots \ldots 2631$

Severin, T. . . .............. 2397

Stamm, H. . . . . . . . . . . . 2678

Storch, $W . \ldots \ldots \ldots \ldots \ldots \ldots \ldots 2517$

Sundermeyer, $W \ldots \ldots \ldots \ldots \ldots \ldots 2458$

Sustmann, R. .............. 2531

Vahrenkamp, H. ........... 2566

Wegner, P. . . . . . . . . . . 2616

Wiehl, W. ............... 2668

Winkhaus, $V . \ldots \ldots \ldots \ldots \ldots \ldots . \ldots 2466$

Würthwein, E.-U. ........... 2444

Zuckerman, J. J. . . . . . . . . . 2656 


\title{
Relative Reaktivität Alkyl-substituierter Alkene und Cycloalkene gegenuiber Diarylcarbenium-Ionen
}

\author{
Herbert Mayr* und Rudolf Pock \\ Institut für Chemie der Medizinischen Universität zu Lübeck, \\ Ratzeburger Allee 160, D-2400 Lübeck 1
}

Eingegangen am 17. Februar 1986

Nach der Konkurrenzmethode wurden die relativen Reaktivitäten Alkyl-substituierter Alkene 4 gegenüber Diarylmethyl-Kationen 2 bestimmt, die in Gegenwart von Alken-Gemischen aus Diarylmethylchloriden 1 und Lewis-Säuren erzeugt wurden. Die Konkurrenzkonstanten werden durch die Art der Lewis-Säure nur wenig beeinflußt, so daß eine eventuelle Differenz der freien Solvatationsenthalpie verschiedener aktivierter Komplexe von der Natur des Gegenions unabhängig sein muß. Aus der 6-50fachen Reaktionsbeschleunigung durch Methylgruppen, die sich am angegriffenen Alken-Kohlenstoff befinden und der $10^{4}$ fachen Reaktivitätssteigerung durch Methylgruppen am neuen Carbeniumzentrum wird auf einen wenig verbrückten Übergangszustand geschlossen.

\section{Relative Reactivities of Alkyl-Substituted Alkenes and Cycloalkenes towards Diarylcarbenium Ions}

The relative reactivities of alkyl-substituted alkenes 4 towards diarylmethyl cations 2 generated in situ from diarylmethyl chlorides 1 and Lewis acids were determined by competition experiments. The relative reactivities were almost independent of the nature of the Lewis acid. Eventual differences of the solvation free enthalpies of various activated complexes are, therefore, independent of the nature of the gegen ions. The rate acceleration by methyl groups $-6-50$ by $\mathrm{CH}_{3}$ at the attacked vinylic position and approximately $10^{4}$ at the developing carbenium centre - indicates a scarcely bridged transition state.

Elektrophile Reaktionen mit Alkenen zählen zu den am eingehendsten untersuchten Reaktionen der organischen Chemie ${ }^{1)}$. Für Halogenierungen, Hydroborierungen, Oxymercurierungen, Arylsulfenylierungen, Carben-Additionen und Säure-katalysierte Hydratisierungen wurden Geschwindigkeitskonstanten oder relative Reaktivitäten verschiedener Alkene bestimmt $^{1 b)}$. Mechanistisch wenig untersucht sind dagegen die zur CC-Verknüpfung nutzbaren Additionen von Carbenium-Ionen an Alkene. Von vereinzelten Beispielen abgesehen ${ }^{2)}$, stammen die derzeitigen Informationen über Alken-Reaktivitäten gegenüber Carbenium-Ionen ausschließlich aus Untersuchungen von carbokationischen Copolymerisationen ${ }^{3)}$. Da die Kenntnis solcher Daten nicht nur für die makromolekulare Chemie ${ }^{4)}$, sondern auch für die Planung organischer Synthesen über aliphatische Friedel-Crafts-Reaktionen von Bedeutung ist $^{5}$, haben wir eine systematische kinetische Untersuchung der Additionen von CarbeniumIonen an Alkene begonnen. Wir berichten hier über relative Reaktivitäten Alkyl-substituierter Alkene und Cycloalkene gegenüber Arylcarbenium-Ionen ${ }^{6}$. 


\section{Reaktionsprodukte}

Diphenylmethylchlorid (1') und sein Dimethylderivat $\mathbf{1}^{\prime \prime}$ eignen sich wegen ihrer hohen Ionisierungstendenz gut zur elektrophilen Alkylierung von Alkenen. In Übereinstimmung mit früheren Voraussagen ${ }^{7}$ bzw. Untersuchungen ${ }^{5,8)}$ liefern die Zinkchlorid/Ether-katalysierten ${ }^{9)}$ Umsetzungen von $1^{\prime}$ und $1^{\prime \prime}$ mit den Alkenen

Schema 1<smiles>[X]c1ccc([CH+]c2ccc([X])cc2)cc1</smiles>

1

$\mathrm{X}=\mathrm{H}: \quad \boldsymbol{1}^{*}-10^{\circ}$

$\mathrm{X}=\mathrm{CH}_{3}: 1 *-10 *$

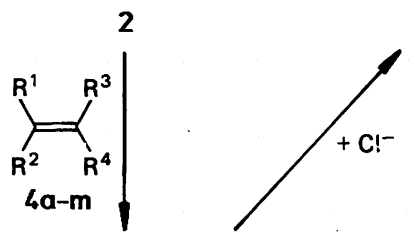

3<smiles>[X]c1ccc(C(c2ccc([X])cc2)C([R7])C([R7])([R7])[Y9]([H])([H])C)cc1</smiles>

5

$\int-\mathrm{H}^{+}$

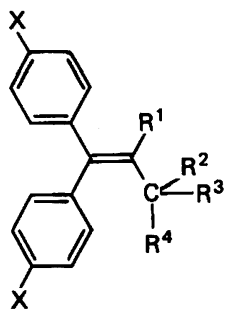

8<smiles>C=C(C)C(C)C(c1ccccc1)c1ccccc1</smiles>

9

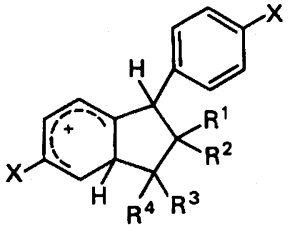

7 für $g^{\circ} \mid-H^{+}$<smiles>[X]c1ccc(C2c3ccc([X])cc3C([R7])([R7])C2([R])[R])cc1</smiles>

10

Chem. Ber. 119 (1986) 
Tab. 1. Reaktionsprodukte der $\mathrm{ZnCl}_{2}$-katalysierten Umsetzung der Diarylmethylchloride $\mathbf{1}^{\prime}$ und 1" mit verschiedenen Alkenen
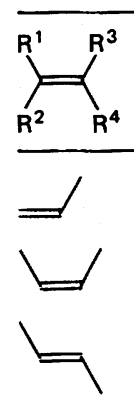

$=1$

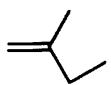<smiles>C=C(C)CCC</smiles><smiles>CC=C(C)C</smiles><smiles>CC(C)=C(C)C</smiles><smiles>C=C1CCC1</smiles><smiles>C=C1CCCC1</smiles><smiles>C=C1CCCCC1</smiles><smiles>CC1=CCCCC1</smiles><smiles>C1=CC2CCC1C2</smiles>

$4 a$

46

$4 c$

$4 d$

$4 \mathrm{e}$

46

49

$3 g^{\circ}(89)^{d \prime}$

$4 h$

$4 i$

$4 j$

$4 \mathbf{k}$

41

$4 \mathrm{~m}$

$3 e^{*}(89)$

$3 f^{\prime}(96)^{c)}$

41

$11(98)^{\text {f) }}$
Produkte (\% Ausb.)

mit $\mathbf{r}^{\circ}$

$3 a^{\circ}(92)^{a)}, 8 a^{\circ}$ (Spur)

$3 b^{\circ}(41), 8 b^{\circ}(8)^{b)}$

$3 c^{\circ}(68), 8 b^{\circ}(10)^{b)}$

$3 h^{\circ}(33), 10 h^{\circ}(60)^{e)}$
$3 d^{\circ}(97)^{\text {al }}$

Produkte (\% Ausb.)

mit 1"*

$3 a^{\prime \prime}(34), 8 a^{*}(10), 10 a^{*}(14)$

$3 b \cdots(61), 8 b \cdots(6)^{b)}$

$3 c^{*}(90), 8 b^{\prime \prime}(5)^{b)}$

3d“ (95)

$3 e^{* *}(91)$

$3 f^{\prime \prime}(91)$

$3 g^{*}(68), 10 g^{*}(10)$

$3 i^{*}(87)$

$3 j^{* *}(82)$

$3 k^{*}(87)$

$31^{*}(84)$

$11^{*}(98)^{f)}$

a) Lit. ${ }^{8)}$. - ${ }^{\text {b) }}$ Diese Reaktionen verlaufen nicht vollständig stereospezifisch und liefern neben 8 noch weitere Produkte in geringen Mengen. Uber Details wird gesondert berichtet ${ }^{10}$. c) Lit. ${ }^{9}$. - d) Bei höherer Konzentration an $\mathrm{ZnCl}_{2} / \mathrm{Et}_{2} \mathrm{O}$ entsteht neben $\mathbf{3 g}$ auch $9 \mathrm{~g}^{\prime}$ und wenig 10g'. - e) Produktverhältnis von den Reaktionsbedingungen abhängig. - ${ }^{0}$ Lit. $^{11)}$.

Chem. Ber. 119 (1986) 
4a-m in hohen Ausbeuten 1:1-Produkte (Tab. 1), weil die Addukte als sekundäre oder tertiäre Alkylhalogenide langsamer ionisieren als die Reaktanten 1' und 1".

Die Bildung der Hauptprodukte 3 erfolgt nach Schema 1 durch Anlagerung eines Chlorid-Ions an die Kationen 6, die bei der Addition der DiarylcarbeniumIonen 2 an die Alkene 4 zunächst entstehen. Bei der Reaktion mit den cis,transisomeren Butenen $\mathbf{4 b}$ und $\mathbf{4 c}$ erhält man vorwiegend in einer anti-Addition die Diastereomeren $\mathbf{3 b}$ und $3 \mathbf{c}$, worüber an anderer Stelle detailliert berichtet wird ${ }^{10}$. Ist 6 ein sekundäres Carbenium-Ion (a-c), erfolgt meist in gewissem Ausmaß eine 1,2-H-Verschiebung unter Bildung von 5 , woraus durch Deprotonierung die 1,1Diarylethylen-Derivate 8 hervorgehen.

Sind $R^{1}$ und $R^{2}$ Methylgruppen, begünstigt der gem-Dialkyl-Effekt ${ }^{12)}$ die Cyclisierung zum Indan: $\mathbf{1 0} \mathbf{h}^{\prime}$ entsteht als Hauptprodukt der Umsetzung von $\mathbf{1}^{\prime}$ mit Tetramethylethylen $(4 \mathrm{~h})$. Im Fall des Trimethylethylen-Addukts $6 \mathbf{g}^{\prime}\left(\mathbf{R}^{1}=\mathrm{CH}_{3}\right.$, $\mathbf{R}^{2}=\mathbf{H}$ ) ist die Cyclisierungstendenz geringer: Neben dem normalen Additionsprodukt $\mathbf{3} \mathrm{g}^{\prime}$ erhält man geringe Mengen des Indans $10 \mathrm{~g}^{\prime}$ sowie eine Spur des terminalen Olefins 9 . Die Umsetzung von Norbornen liefert, wie kürzlich beschrieben ${ }^{11)}$, exo-2-Chlor-syn- und -anti-7-alkylierte Norbornane, die sich unter den hier beschriebenen HPLC-Bedingungen nicht voneinander trennen lassen und daher im folgenden als einheitliches Produkt 11' bzw. 11" betrachtet werden.



\section{Irreversibilität der Additionsreaktionen}

Da die relativen Alken-Reaktivitäten nach der Konkurrenzmethode bestimmt werden sollten, mußte sichergestellt sein, daß die Additionsreaktionen irreversibel ablaufen, weil nur dann aus dem Verhältnis der Additionsprodukte Rückschlüsse auf die relativen Additionskonstanten gezogen werden können.

Daher wurde 3d', das Additionsprodukt von Diphenylmethylchlorid (1') an Isobuten (4d), in Gegenwart von $\mathrm{ZnCl}_{2}$ mit überschüssigem Trimethylethylen (4g) behandelt (Schema 2). Das Addukt 3d' blieb hierbei unverändert. Entsprechend führte auch die Behandlung von 3 $\mathbf{g}^{\prime}$ mit Isobuten unter den Bedingungen der Additionsreaktion nicht zum Entstehen von 3d'.

Schema 2

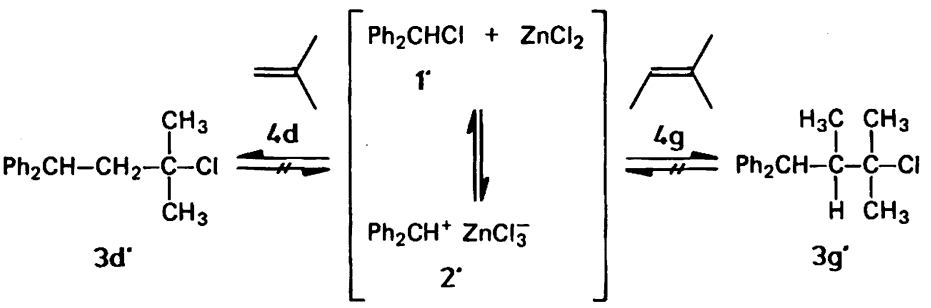

Chem. Ber. 119 (1986) 
Das in Schema 2 beschriebene Experiment schließt eine Rückspaltung der Additionsprodukte $\mathbf{3}$ in $\mathbf{2}$ und $\mathbf{4}$ unter den hier gewählten Reaktionsbedingungen aus. Daraus folgt jedoch nicht zwingend die Irreversibilität des Additionsschrittes; eine reversible Bildung von 6 aus 2 und 4, der sich eine irreversible Chloridanlagerung unter Bildung von 3 anschließt, wäre mit dieser Beobachtung ebenfalls vereinbar. Diese Möglichkeit wurde durch das in Schema 3 skizzierte Kreuzungsexperiment ausgeschlossen.

\section{Schema 3}

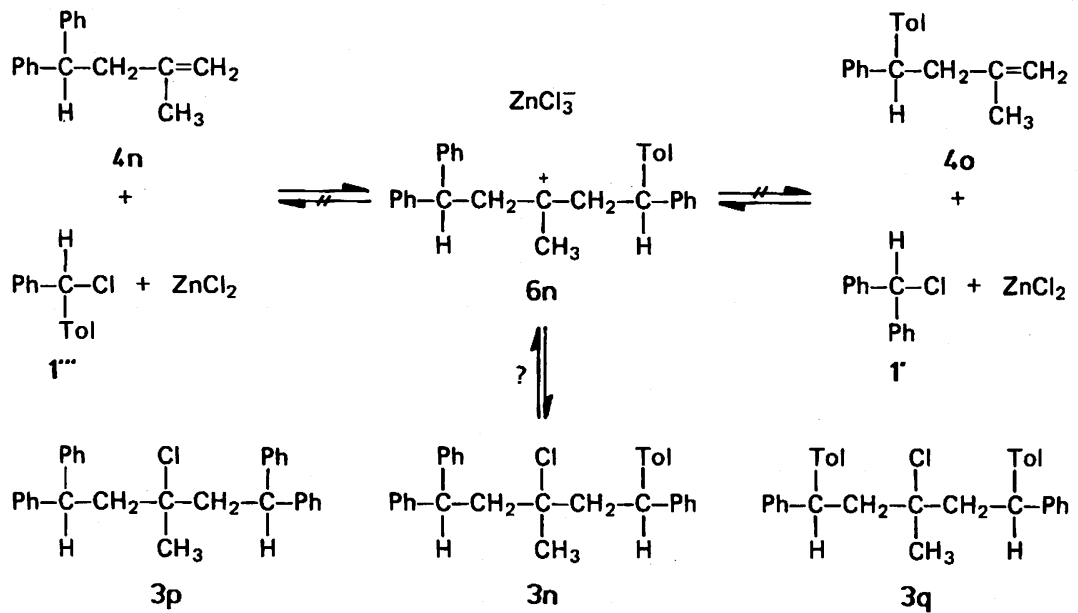

Setzt man das aus $3 \mathbf{d}^{\prime}$ zugängliche Alken $4 \mathrm{n}$ unter den üblichen Additionsbedingungen mit Phenyltolylmethylchlorid ( $\left.\mathbf{1}^{\prime \prime \prime}\right)$ um, erhält man ausschließlich das Additionsprodukt 3n mit einer Tolyl- und drei Phenylgruppen. Dasselbe Additionsprodukt erhält man in gleicher Weise aus dem Alken 40 und $\mathbf{1}^{\prime}$. Wäre die Bildung von 6n aus 40 und $1^{\prime}$ reversibel, müßte 6n parallel zur Rückreaktion auch eine Spaltung in 4n und $\mathbf{1}^{\prime \prime \prime}$ eingehen. Man würde ein Produktgemisch erwarten, das außer 3n noch 3p und 3q, die Kopplungsprodukte aus $\mathbf{1}^{\prime}$ und 4n bzw. 1"' und 4o, enthält. Im Massenspektrum des ölig anfallenden Additionsproduktes $3 \mathbf{n}$ fehlten jedoch charakteristische Signale der unabhängig synthetisierten Verbindungen 3p und 3q, so daß die Spaltung von 6n ausgeschlossen werden kann. Während für die anderen Kationen des Typs 6, die im Rahmen dieser Studie auftreten, wegen ihrer ähnlichen Struktur ein entsprechendes Verhalten erwartet werden kann, muß bei Additionen stark dotierter Diarylcarbenium-Ionen die oben diskutierte Rückreaktion in Betracht gezogen werden ${ }^{13}$.

\section{Relative Alken-Reaktivitäten aus Konkurrenzexperimenten}

Um die relative Reaktivität zweier Alkene zu bestimmen, wurden unterschiedlich zusammengesetzte Gemische von zwei Alkenen in Dichlormethan bei -70 bzw.

Chem. Ber. 119 (1986) 
$-78^{\circ} \mathrm{C}$ in Gegenwart von Zinkchlorid/Ether mit einer geringen Menge an $1^{\prime}$ bzw. $1^{\prime \prime}$ versetzt. Dabei erhielt man Gemische von 1:1-Produkten, die mit Hilfe der HPLC-Methode analysiert wurden.

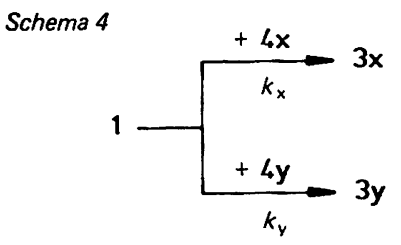

Schema 5



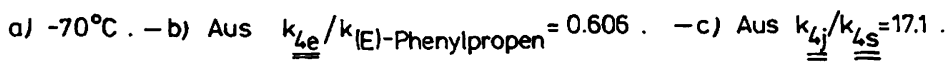

$4 \mathrm{~s} \quad \mathrm{H}_{2} \mathrm{C}=\mathrm{CH}-\mathrm{CH}_{2} \mathrm{Si}\left(\mathrm{CH}_{3}\right)_{3}$

Chem. Ber. 119 (1986) 
Da es im allgemeinen nicht zweckmäßig war, die Alkene in so großem Überschuß einzusetzen, daß ihr Verhältnis während der Reaktion annähernd konstant blieb, wurden die Konkurrenzkonstanten nach Gl. (1) bestimmt, in die außer den Anfangskonzentrationen der Reaktanten nur das Verhältnis der beiden Additionsprodukte eingeht ${ }^{14)}$.

$$
k_{\mathrm{x}} / k_{\mathrm{y}}=\lg \left(1-\frac{[1]_{0} R}{[4 \mathrm{x}]_{0}(1+R)}\right) / \lg \left(1-\frac{[1]_{0}}{[4 \mathrm{y}]_{0}(1+R)}\right) \text { wobei } R=\frac{[3 \mathrm{x}]}{[3 \mathrm{y}]}
$$

Jede Konkurrenzkonstante wurde mehrfach bestimmt, wobei die relativen Alken-Konzentrationen variiert wurden. Dabei erhielt man die in Schema 5 wiedergegebenen Konkurrenzkonstanten mit durchschnittlichen Standardabweichungen von $\pm 4.3 \%$. Da hierbei systematische Fehler unentdeckt bleiben, wurde in vielen Fällen die relative Reaktivität eines Olefin-Paars sowohl direkt wie auch durch Vergleich mit einem dritten Alken bestimmt. Die auf verschiedenen Wegen ermittelten relativen Geschwindigkeitskonstanten unterscheiden sich um durchschnittlich $\pm 6 \%$.

Die hier und in Lit. ${ }^{15,16)}$ angegebenen Geschwindigkeitsverhältnisse wurden logarithmiert, wobei man ein überbestimmtes lineares Gleichungssystem erhielt, das nach der Methode der kleinsten Fehlerquadrate gelöst wurde. Die auf diese Weise gemittelten $k_{\text {rel- }}$-Werte sind in Schema 5 angegeben.

In Tab. 2 werden die mit verschiedenen Katalysatoren bestimmten relativen Reaktivitäten von 2-Methyl-1-penten (4f) und Norbornen (4 m) gegenüber dem Diphenylcarbenium-Ion verglichen. Die relativ geringen Unterschiede dieser Werte deuten an, daß eventuelle Differenzen der freien Solvatationsenthalpie verschiedener aktivierter Komplexe von der Natur des Gegenions kaum beeinflußt werden. Dadurch läßt sich eine spezifische Kation-Anion-Wechselwirkung ausschließen, insbesondere daß im Übergangszustand die Ausbildung der neuen Kohlenstoff-Halogen-Bindung bereits eingesetzt hat. Es ist jedoch denkbar, daß die Konkurrenzkonstante von der Art des Anions abhängig wird, wenn bei der Addition weniger stabilisierte Carbenium-Ionen entstehen.

Tab. 2. Abhängigkeit der Konkurrenzkonstanten von Art und Menge der Lewis-Säure bei $-78^{\circ} \mathrm{C}$

\begin{tabular}{llcll}
\hline I' $^{\prime}(\mathrm{mmol})$ & $4 \mathrm{ff}(\mathrm{mmol})$ & $\mathbf{4 m}(\mathrm{mmol})$ & Lewis-Säure $(\mathrm{mmol})$ & \multicolumn{1}{c}{$k_{\mathbf{4 f}} / k_{\mathbf{4 m}}$} \\
\hline 0.51 & $1.1-1.2$ & $16-20$ & $\mathrm{ZnCl}_{2}(1.9) / \mathrm{Et}_{2} \mathrm{O}(3.0)$ & $21.4 \pm 0.07(2)$ \\
$0.48-0.58$ & $0.69-0.84$ & $8.7-27.3$ & $\mathrm{BCl}_{3}(0.95)$ & $18.5 \pm 0.9(3)$ \\
$0.38-0.63$ & $0.74-0.90$ & $9.1-27.4$ & $\mathrm{SnCl}_{4}(0.85)$ & $18.4 \pm 0.4(3)$ \\
\hline
\end{tabular}

Besitzt ein Molekül mehrere Mehrfachbindungen, liegt eine intramolekulare Konkurrenz-Situation vor. Limonen (4r) kann mit 1" an der exo- oder endocyclischen Doppelbindung reagieren, wobei die Produkte 3ra und 3rb (Konfiguration unklar!) unter $\mathrm{ZnCl}_{2} / \mathrm{Et}_{2} \mathrm{O}$ - oder $\mathrm{SnCl}_{4}$-Katalyse im Verhältnis 2.5:1 entstehen. Da dieses Verhältnis annähernd dem Reaktionsverhältnis $4 \mathrm{f} / 41$ entspricht, zeigt es sich, daß die Konkurrenzdaten in Schema 5 zur Vorhersage der Position des elektrophilen Angriffs an mehrfach ungesättigten Systemen genutzt werden können. 


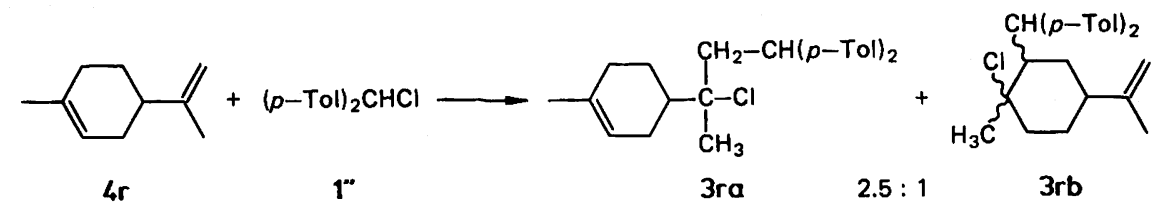

\section{Diskussion}

Schema 5 zeigt, daß die Reaktivitätsabstufung der Alkene 4a-m gegenüber den beiden Elektrophilen $2^{\prime}$ und $2^{\prime \prime}$ ähnlich ist. Aus dem Reaktivitätsverhältnis der beiden Alkene 4f/4m gegenüber $\mathbf{2}^{\prime}$ (21.4) und 2" (27.7) und dem Verhältnis 4m/4a gegenüber 2' (304) und 2" (1152) ließe sich eine höhere Selektivität des weniger reaktiven Carbenium-Ions $2^{\prime \prime}$ ableiten. Diese Beziehung gilt jedoch nicht allgemein, da sich bei den Vergleichen $\mathbf{4 g} / \mathbf{4 f}$ und $4 \mathrm{c} / \mathbf{4 a}$ das reaktivere CarbeniumIon $2^{\prime}$ als selektiver erweist. Das hierbei zutage tretende inverse ReaktivitätsSelektivitäts-Verhalten scheint dadurch bedingt zu sein, daß Methylgruppen am angegriffenen Zentrum des Alkens die Additionsbereitschaft gegenüber dem reaktiveren Carbenium-Ion stärker fördern, was auch bei der Einführung von $\beta$-Methylgruppen in Styrol gefunden wurde ${ }^{15}$. Da das derzeit verfügbare Material für eine fundierte Reaktivitäts-Selektivitäts-Diskussion ${ }^{17}$ noch nicht ausreicht, wollen wir hier auf die Unterschiede bei der Wahl von 1' bzw. 1" als Bezugssystem noch nicht eingehen.

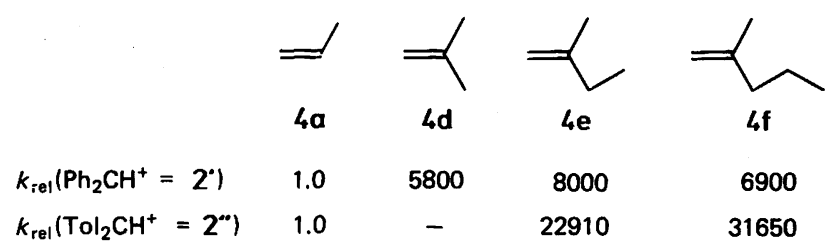

Erwartungsgemäß unterscheiden sich die 1,1-dialkylierten Alkene 4d,e,f nur wenig in ihrer Reaktivität gegenüber Diarylcarbenium-Ionen. Sie sind etwa $10^{4} \mathrm{mal}$ reaktiver als Propen (4a), einem $\Delta \Delta G^{*}$. Wert von $3.5-4.2 \mathrm{kcal} / \mathrm{mol}\left(-70^{\circ} \mathrm{C}\right)$ entsprechend.

Arnett berichtete, daß die Ionisierung von Isopropylchlorid $\left[\rightarrow\left(\mathrm{CH}_{3}\right)_{2} \mathrm{CH}^{+}\right]$ mit Antimonpentafluorid in $\mathrm{SO}_{2} \mathrm{ClF}$ um $9.5 \mathrm{kcal} / \mathrm{mol}$ weniger exotherm ist als die von tert-Butylchlorid ${ }^{18,19}$. Daraus läßt sich in Kombination mit den Bildungswärmen ${ }^{20)}$ von Isopropylchlorid, tert-Butylchlorid, Propen und Isobuten abschätzen, daß die Bildung eines tertiären Carbenium-Ions aus Isobuten in $\mathrm{SO}_{2} \mathrm{ClF}$ um $9.2 \mathrm{kcal} / \mathrm{mol}$ stärker exotherm sein sollte als die Bildung eines sekundären Carbenium-Ions aus Propen (Abb. 1). Nach der gleichen Methode ermittelt man aus den Ionisierungswärmen in Dichlormethan ${ }^{18)}$, daß sich in diesem Lösungsmittel

Chem. Ber. 119 (1986) 
der Unterschied dieser relativen Protonierungswärmen auf $7.3 \mathrm{kcal} / \mathrm{mol}$ verkleinert. Unter der Annahme konstanter Entropie-Unterschiede ergibt sich daraus, $\mathrm{da} \mathrm{B}$ im Übergangszustand der hier untersuchten Additionsreaktionen die unterschiedliche Stabilisierung der entstehenden Carbenium-Ionen zu etwa 50\% ausgeprägt ist.

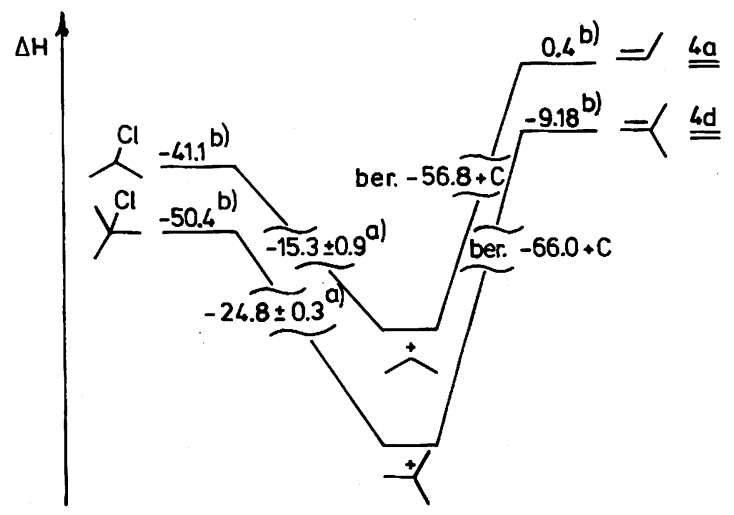

Abb. 1. Abschätzung der relativen Reaktionsenthalpie der Protonierung von Isobuten und Propen in $\mathrm{SO}_{2} \mathrm{ClF}(\mathrm{kcal} / \mathrm{mol})$

a) $\Delta H$ der Reaktion $\mathrm{R}-\mathrm{Cl}+\mathrm{SbF}_{5} \rightarrow \mathrm{R}^{+}$in $\mathrm{SO}_{2} \mathrm{ClF}$ : Lit. ${ }^{18,19)}$; b) $\Delta H_{\mathrm{f}}^{\circ}$ (fl.): Lit. $^{20)}$

Methylencyclobutan (4i) und Methylencyclohexan (4k) unterscheiden sich in ihrer Reaktivität nur wenig von 2-Methyl-1-penten (4f). Dieser Befund ist nicht überraschend, da es sich jeweils um 1,1-dialkylsubstituierte Alkene handelt. Außerdem sollten Ringspannungseffekte keine Rolle spielen, da die Hybridisierung der Ringkohlenstoffe beim elektrophilen Angriff nicht verändert wird. Obwohl diese Argumente in gleicher Weise auch für Methylencyclopentan (4j) gelten, ist dieses Olefin deutlich reaktiver.

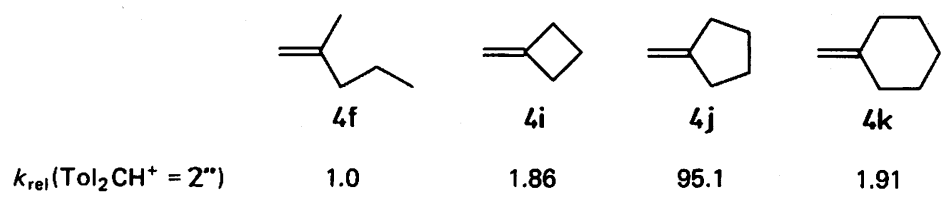

Die ungewöhnlich rasche Bildung von Cyclopentyl-Kationen wurde wiederholt beim Vergleich der Solvolysegeschwindigkeiten von Cyclopentyl-Derivaten mit Cyclohexyl und entsprechenden acyclischen Verbindungen beobachtet (Tab. 3) und durch den Wegfall ekliptischer Wechselwirkungen bei der Heterolyse des Fünfring-Derivats erklärt ${ }^{21)}$.

Diese Deutung ist jedoch fragwürdig, da Arnett annähernd gleiche Ionisierungswärmen für 1-Chlor-1-methylcyclopentan, 1-Chlor-1-methylcyclohexan und 2-Chlor-2-methylbutan fand ${ }^{19)}$, obwohl diese Daten ebenfalls den Abbau an Spannung beim Fünfring wiedergeben müßten. 
Tab. 3. Vergleich der Solvolysegeschwindigkeiten von Isopropyl-, Cyclopentyl- und Cyclohexyl-Derivaten ${ }^{21)}$

\begin{tabular}{lllccc}
\hline $\mathbf{R}$ & $\mathbf{X}$ & Reaktionsbedingungen & & & \\
& & & & \\
& & & & \\
$\mathrm{CH}_{3}$ & $\mathrm{Cl}$ & $80 \%$ Ethanol, $25^{\circ} \mathrm{C}$ & 1.00 & 44 & 0.35 \\
$\mathrm{H}$ & $\mathrm{Cl}$ & $50 \%$ Ethanol, $95^{\circ} \mathrm{C}$ & 1.00 & 5.2 & 0.36 \\
$\mathrm{H}$ & $\mathrm{OTs}$ & Essigsäure, $50^{\circ} \mathrm{C}$ & 1.00 & 18 & 0.88 \\
\hline
\end{tabular}

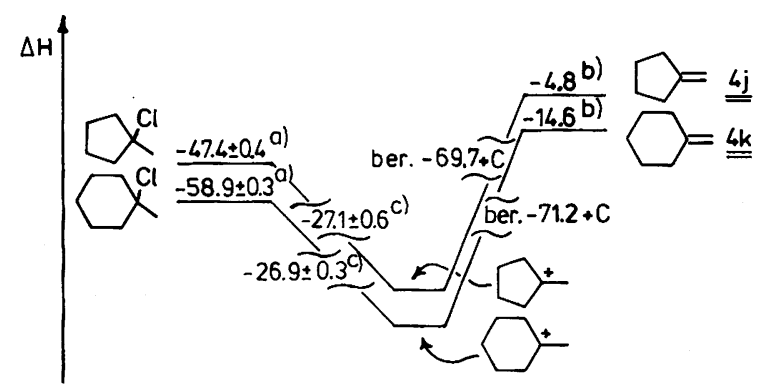

Abb. 2. Abschätzung der relativen Reaktionsenthalpie der Protonierung von Methylencyclopentan (4j) und Methylencyclohexan (4k) in $\mathrm{SO}_{2} \mathrm{ClF}(\mathrm{kcal} / \mathrm{mol})$.

a) $\Delta H_{\mathrm{f}}^{\circ}$ (fl.): Lit. ${ }^{\text {9); }}$ b) $\Delta H_{\mathrm{f}}^{\circ}$ (fl.): Lit. ${ }^{20)}$; c) $\Delta H$ der Reaktion $\mathrm{R}-\mathrm{Cl}+\mathrm{SbF}_{5} \rightarrow \mathrm{R}^{+}$in $\mathrm{SO}_{2} \mathrm{ClF}$ : Lit. $^{19)}$

Aus Arnetts Ionisierungswärmen lassen sich nach Abb. 2 relative Protonierungswärmen für $\mathbf{4 j}$ und $\mathbf{4 k}$ abschätzen, die im Rahmen der Meßgenauigkeit übereinstimmen. Aus den experimentell bestimmten Protonenaffinitäten von Methylcyclopenten und Methylcyclohexen ${ }^{22 a)}$ lassen sich unter Berücksichtigung der verschiedenen Grundzustandsenergien ${ }^{22 b}$ ) für $4 \mathbf{j}$ und $\mathbf{4 k}$ gleicheProtonenaffinitäten ableiten. Damit übereinstimmend wurden für die Protonierung von Cyclopentanon $(-17.6 \mathrm{kcal} / \mathrm{mol})$ und Cyclohexanon $(-18.2 \mathrm{kcal} / \mathrm{mol})$ in Fluorsulfonsäure nahezu gleiche Reaktionswärmen gefunden ${ }^{22)}$. Schließlich läßt sich auch in den ${ }^{13}$ C-NMR-Spektren 1-Aryl-substituierter Cyclopentyl- und Cyclohexyl-Derivate durch das ,tool of increasing electron demand" kein Hinweis auf eine unterschiedliche Stabilisierung der positiven Ladung in Fünf- und Sechsring finden ${ }^{23}$.

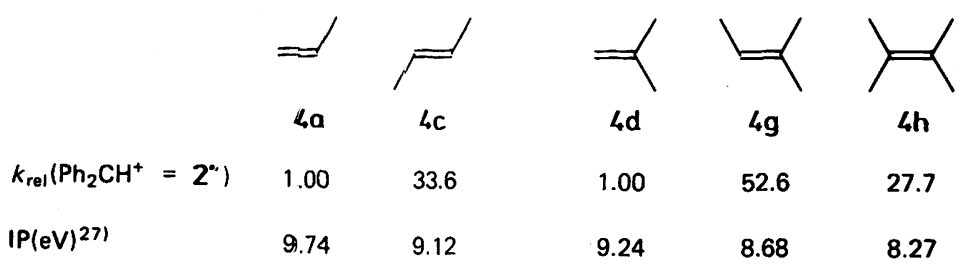

Chem. Ber. 119 (1986) 
Dennoch wird Methylencyclopentan (4j) 50mal rascher als $4 \mathrm{k}$ durch $\mathbf{2}^{\prime \prime}$ angegriffen, und auch bei den elektrophilen Additionen von $\operatorname{ISCN}\left(k_{4 \mathrm{j}}: k_{4 \mathrm{k}}=2.1\right), 2,4-$ $\left(\mathrm{NO}_{2}\right)_{2} \mathrm{C}_{6} \mathrm{H}_{3} \mathrm{SCl}(3.1)$ und $\mathrm{NOCl}(5.3)^{24)}$ sowie von Dichlorcarben $(2.4)^{25)}$ erweist sich $4 \mathrm{j}$ reaktiver als $4 \mathbf{k}$. Da auch die Ionisierungspotentiale dieser beiden Alkene kein eindeutiges Indiz für die höhere Reaktivität von $4 \mathbf{j}$ ergeben $(4 \mathrm{j}: 9.15 \mathrm{eV}, \mathbf{4 k}$ : $9.13 \mathrm{eV})^{26)}$, müssen wir den Grund für diesen kinetischen Effekt ebenso offen lassen wie die Ursache für die rasche Solvolyse der Cyclopentyl-Derivate.

trans-2-Buten reagiert mit Diarylmethyl-Kationen um etwa eine Zehnerpotenz rascher als Propen. Vernachlässigt man die unterschiedliche Zahl an gauche-Wechselwirkungen in den Additionsprodukten an Propen und trans-Buten, läßt sich aus Abb. 3 entnehmen, daß die elektrophile Addition an Propen um etwa $3 \mathrm{kcal} /$ mol stärker exotherm sein sollte als die entsprechende Reaktion mit trans-Buten, woraus man die umgekehrte Reaktivitätsabstufung ableiten würde.
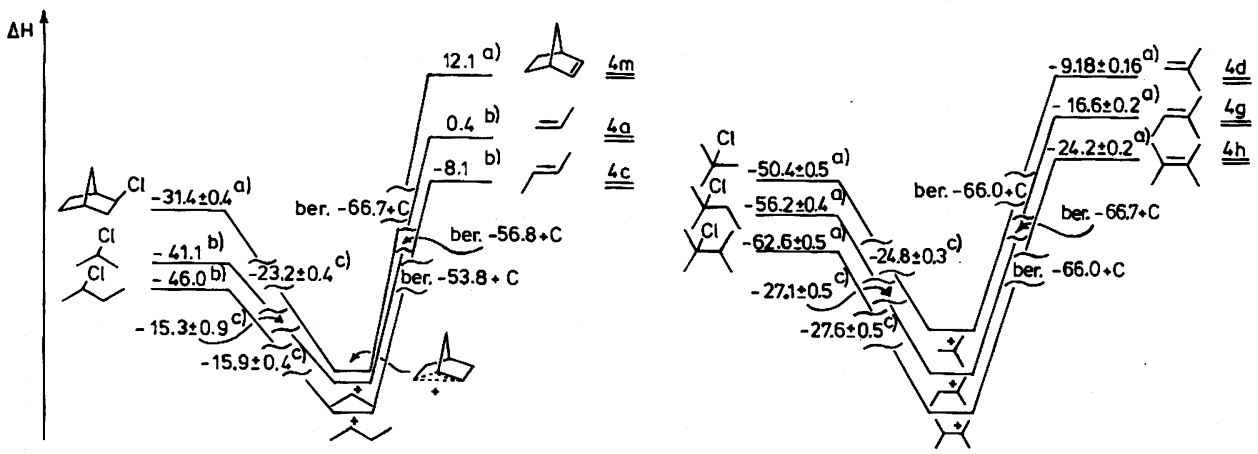

Abb. 3. Einfluß von $\beta$-Methyl-Substituenten $(\mathrm{kcal} / \mathrm{mol})$ auf die relativen Protonierungswärmen von Alkenen in $\mathrm{SO}_{2} \mathrm{ClF}$.

a) $\Delta H_{\text {f }}^{\circ}$ (fl.): Lit. ${ }^{19)}$; b) $\Delta H_{\mathrm{f}}^{\circ}$ (fl.): Lit. ${ }^{20)}$; c) $\Delta H$ der Reaktion $\mathrm{R}-\mathrm{Cl}+\mathrm{SbF}_{5} \rightarrow \mathrm{R}^{+}$in $\mathrm{SO}_{2} \mathrm{ClF}$ : Lit. ${ }^{19)}$

Die Einführung einer $\beta$-Methylgruppe in Isobuten $(\mathbf{4 d} \rightarrow \mathbf{4 g})$ bewirkt eine noch deutlichere Reaktivitätssteigerung, während die zweite $\beta$-Methylgruppe $(\rightarrow \mathbf{4 h})$ eine geringfügige Verlangsamung verursacht. Abb. 3 zeigt, daß Protonenanlagerungen an diese drei Alkene zu vergleichbaren Wärmetönungen führen. Wir nehmen daher an, daß ein partiell verbrückter Übergangszustand bzw. die Anhebung des HOMO für den Reaktivitätsanstieg von $\mathbf{4 a} \rightarrow \mathbf{4 c}$ und $\mathbf{4 d} \rightarrow \mathbf{4 g}$ verantwortlich ist. Die gleichzeitig erhöhte sterische Repulsion wird dadurch überkompensiert. Erst bei der Einführung der zweiten $\beta$-Methylgruppe $(\rightarrow 4 \mathrm{~h})$ wirkt sich die sterische Reaktionsverzögerung etwas stärker aus als die elektronische Beschleunigung.

Aufgrund der besonderen Stabilisierung des Norbornyl-Kations und der gespannten Doppelbindung in Norbornen ergibt sich für $\mathbf{4 m}$ nach Abb. 3 eine relative Protonierungswärme, die der von 1,1-dialkylierten Alkenen entspricht. Die Geschwindigkeit der Carbenium-Additionen an Norbornen wie auch die der entsprechenden Protonierung ${ }^{28}$, die zwischen der von 1,2- und 1,1-dialkylierten Ethylenen liegt, ist daher geringer als aufgrund der thermodynamischen Überlegungen erwartet. Die viel diskutierte ungewöhnlich hohe Reaktivität von Norbornen, die

Chem. Ber. 119 (1986) 
bei Cycloadditionen ${ }^{29)}$ und auch bei Additionen einiger Elektrophile (Tab. 4) beobachtet wird, könnte auf Reaktionen mit cyclischem Übergangszustand beschränkt sein.

\section{Vergleich mit anderen elektrophilen Additionen}

Für den Angriff eines Elektrophils an eine CC-Doppelbindung sind zwei extreme Situationen denkbar (Schema 6): a) Das Elektrophil nähert sich einem Ende der Doppelbindung, so daß ein unverbrücktes Carbenium-Ion entsteht. b) Der elektrophile Angriff erfolgt auf die Mitte des $\pi$-Systems, wobei ein symmetrisch verbrücktes Kation erhalten wird.

Schema 6
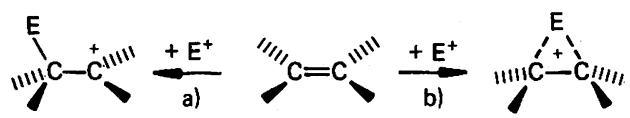

Melloni, Modena und Tonellato zeigten, daß Elektrophile der Kategorie a) mit Alkenen und Alkinen ähnlich rasch reagieren, während Elektrophile der Kategorie b) gegenüber Alkenen deutlich reaktiver sind als gegenüber Alkinen ${ }^{30)}$. CarbeniumIonen wurden, wie Proton-Additionen, in Kategorie a) eingeordnet, da sie gegenüber Alkenen und entsprechend substituierten Alkinen ähnliche Reaktivität zeigten. Ruasse, Dubois und Argile verwendeten als Kriterium zur Unterscheidung cyclischer und acyclischer Übergangszustände bei Bromierungen, Sulfenylierungen und Hydratisierungen den Einfluß von Methylgruppen auf die Reaktionsgeschwindigkeit ${ }^{31)}$. Zur Anwendung dieses mechanistischen Kriteriums auf die Additionen von Carbenium-Ionen werden in Tab. 4 die hier bestimmten relativen Reaktivitäten anderen elektrophilen Additionen gegenübergestellt.

Tab. 4 zeigt charakteristische Reaktivitätsunterschiede zwischen monoalkylierten sowie 1,2- und 1,1-dialkylierten Alkenen: Während bei Hydratisierungen die Reaktivitätssteigerung von Propen zu trans-2-Buten 15 beträgt, beträgt sie $\mathrm{zu}$ Isobuten 5 Zehnerpotenzen, in Einklang mit einem nicht verbrückten Übergangszustand. Im Gegensatz dazu sind trans-Buten und Isobuten gegenüber $\mathrm{Cl}_{2}$ und $\mathrm{Br}_{2}$ etwa 50mal reaktiver als Propen; dies ist für einen verbrückten Übergangszustand $\mathrm{zu}$ erwarten, bei dem beide ehemaligen $\mathrm{sp}^{2}$-Kohlenstoffe einen Teil der positiven Ladung tragen (Halonium-Ionen-Charakter). Methyl-Effekte wie bei Halogenierungen wurden auch bei den Additionen von Nitrosylchlorid gefunden, für die ein viergliedriger Ring als Übergangszustand diskutiert wurde. Deutlich schwächer ausgeprägt sind die Methyl-Effekte bei den in Tab. 4 angeführten Sulfenylierungen, Selenylierungen und Quecksilberacetat-Additionen, bei denen der Alken-Teil im Übergangszustand kaum positive Ladung übernommen hat.

Der Vergleich der Substituenten-Effekte in Tab. 4 zeigt deutlich, daß Carbenium-Ionen-Additionen die größte Analogie zu den Säure-katalysierten Hydratisierungen zeigen. Ein Unterschied besteht jedoch darin, daß bei Carbenium-IonenAdditionen Trimethylethylen deutlich reaktiver ist als Isobuten, während bei den Proton-Additionen die zusätzliche Methylgruppe sogar eine geringe Reaktivitätsabnahme verursacht. Die unterschiedliche Grenzorbital-Wechselwirkung, auf die 
Tab. 4. Relative Alken-Reaktivitäten gegenüber verschiedenen Elektrophilen

\begin{tabular}{|c|c|c|c|c|c|c|c|}
\hline & $\mathbf{R}$ & $\Longrightarrow$ & 4c & 4d & 49 & $4 m$ & Lit. \\
\hline $\begin{array}{l}\mathrm{Ph}_{2} \mathrm{CHCl} / \mathrm{ZnCl}_{2}-\mathrm{Et}_{2} \mathrm{O} / \\
\mathrm{CH}_{2} \mathrm{Cl}_{2} /-70 \text { bzw. }-78^{\circ} \mathrm{C}\end{array}$ & $\mathrm{CH}_{3}$ & 1.00 & 33.6 & $5.80 \cdot 10^{3}$ & $3.05 \cdot 10^{5}$ & $3.31 \cdot 10^{2}$ & diese Arbeit \\
\hline $\begin{array}{l}\mathrm{Tol}_{2} \mathrm{CHCl} / \mathrm{ZnCl}_{2}-\mathrm{Et}_{2} \mathrm{O} / \\
\mathrm{CH}_{2} \mathrm{Cl}_{2} /-70^{\circ} \mathrm{C}\end{array}$ & $\mathrm{CH}_{3}$ & 1.00 & 9.27 & $3.17 \cdot 10^{4 a)}$ & $7.63 \cdot 10^{5}$ & $1.15 \cdot 10^{3}$ & diese Arbeit \\
\hline aq. $\mathrm{H}_{2} \mathrm{SO}_{4}, 25^{\circ} \mathrm{C}$ & $\mathrm{CH}_{3}$ & $1.00^{b)}$ & 14.8 & $1.56 \cdot 10^{5}$ & $9.03 \cdot 10^{4}$ & $1.43 \cdot 10^{4 c)}$ & 32) \\
\hline $\mathrm{Cl}_{2}$, ohne Solvens, $-9^{\circ} \mathrm{C}$ & $\mathrm{C}_{2} \mathrm{H}_{5}$ & 1.00 & 50 & 58 & $1.1 \cdot 10^{4}$ & & 33) \\
\hline $\mathrm{Br}_{2}, \mathrm{CH}_{3} \mathrm{OH}, 25^{\circ} \mathrm{C}$ & $\mathrm{CH}_{3}$ & 1.00 & 32.3 & 92.3 & $3.21 \cdot 10^{334)}$ & & 31) \\
\hline $\mathrm{NOCl}, \mathrm{CHCl}_{3}, 0^{\circ} \mathrm{C}$ & $\mathrm{C}_{2} \mathrm{H}_{5}$ & 1.00 & 155 & 47 & $3.5 \cdot 10^{3}$ & $1.3 \cdot 10^{4}$ & 24) \\
\hline $2,4-\left(\mathrm{NO}_{2}\right)_{2} \mathrm{C}_{6} \mathrm{H}_{3} \mathrm{SCl}, \mathrm{CH}_{3} \mathrm{CO}_{2} \mathrm{H}, 30^{\circ} \mathrm{C}$ & $\mathrm{C}_{2} \mathrm{H}_{5}$ & 1.00 & 2.08 & 3.39 & 15.4 & 254 & 24) \\
\hline $\mathrm{C}_{6} \mathrm{H}_{5} \mathrm{SeCl}, \mathrm{CH}_{2} \mathrm{Cl}_{2}, 25^{\circ} \mathrm{C}$ & $\mathrm{CH}_{3}$ & 1.00 & 0.238 & 0.772 & 0.430 & & 35) \\
\hline $\mathrm{Hg}(\mathrm{OAc})_{2}, \mathrm{CH}_{3} \mathrm{OH}$ & $\mathrm{C}_{2} \mathrm{H}_{5}$ & 1.00 & & 1.40 & 0.25 & $0.74^{\mathrm{d})}$ & 36) \\
\hline \multicolumn{8}{|c|}{ 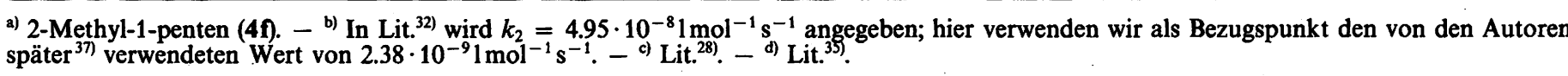 } \\
\hline
\end{tabular}


der Reaktivitätsunterschied $\mathbf{4 d} / \mathbf{4 g}$ gegenüber Carbenium-Ionen zurückgeführt werden kann (Abschnitt 4.), spielt bei der Addition des „harten“ $\mathrm{H}^{+}$keine Rolle. Die ähnlichen relativen Protonierungswärmen von $4 \mathrm{~d}$ und $\mathbf{4 g}$ spiegeln sich daher in den ähnlichen Protonierungsgeschwindigkeiten wider.

Aufgrund der stark unterschiedlichen $\alpha$ - und $\beta$-Methyleffekte lassen sich stark verbrückte Übergangszustände bei Carbenium-Ionen-Additionen an Alkene ausschließen. Eine partielle Verbrückung, wie sie durch die stereochemischen Befunde gefordert wird ${ }^{10)}$, steht jedoch auch mit diesen Ergebnissen in Einklang.

Wir danken Frau M. Rubow für ihre Mitarbeit bei einigen Experimenten, der Deutschen Forschungsgemeinschaft und dem Fonds der Chemischen Industrie für finanzielle Unterstützung.

\section{Experimenteller Teil}

NMR-Spektren: Jeol JNM-C-60-HL, Jeol JNM-PS-100 und Varian XL 200. - Massenspektren: Varian MAT CH 4 und MAT 311 A. - HPLC-Analysen: Orlita MK 00 und Knauer HPLC Pumpe 64; Stahlsäulen mit $250 \mathrm{~mm}$ Länge und $4.6 \mathrm{~mm}$ Innendurchmesser; UV-Detektoren SF 770 Spektroflow (Schoeffel Instr.) und SF 769 Z (Kratos Analyt. Instr.) mit Meßwellenlänge $254 \mathrm{~nm}$.

Da käufliches Diphenylmethylchlorid (1') meist durch Benzophenon verunreinigt ist, erhielt man 1' und 1" durch Einleiten von Chlorwasserstoff in eine Petrolether (Sdp. $40-60^{\circ} \mathrm{C}$-Suspension der entsprechenden Diarylmethanole, die durch Umkristallisieren aus Ethanol gereinigt wurden. Dichlormethan wurde nach 15 stdg. Kochen über $\mathrm{P}_{2} \mathrm{O}_{5}$ destilliert, anschließend 10-15 h über Blei-Natrium-Legierung gekocht und destilliert. Der als Katalysator verwendete Zinkchlorid-Ether-Komplex wurde nach Lit." hergestellt und als Lösung in Dichlormethan gelagert.

\section{Präparative Umsetzungen}

1. Propen (4a) und Diphenylmethylchlorid (1'): Die nach Lit. ${ }^{8)}$ durchgeführte Umsetzung liefert neben 3 $\mathbf{a}^{\prime}$ mitunter geringe Mengen 1,1-Diphenyl-1-buten (8 $\left.\mathbf{a}^{\prime}\right)$. $\mathrm{Zu}$ seiner quantitativen Bestimmung bei den Konkurrenzexperimenten wurde es analog dem 1,1-Diphenylethylen $^{38)}$ aus Butansäure-ethylester und Phenylmagnesiumbromid synthetisiert. - ${ }^{1} \mathbf{H}$ $\operatorname{NMR}\left(\mathrm{CCl}_{4}\right) ; \delta=1.03\left(\mathrm{t}, J=7 \mathrm{~Hz} ; 3 \mathrm{H}, \mathrm{CH}_{3}\right), 2.12\left(\mathrm{~m} ; 2 \mathrm{H}, \mathrm{CH}_{2}\right), 6.00(\mathrm{t}, J=7 \mathrm{~Hz} ; 1 \mathrm{H}$, Vinyl-H), $7.13-7.22(\mathrm{~m} ; 10 \mathrm{H}$, Aromaten-H).

2. Propen (4a) und Bis(4-methylphenyl)methylchlorid ( $\left.1^{\prime \prime}\right): 2.64 \mathrm{~g}(62.7 \mathrm{mmol})$ 4a wurden bei $-78^{\circ} \mathrm{C}$ in eine Lösung von $0.52 \mathrm{~g}(3.8 \mathrm{mmol}) \mathrm{ZnCl}_{2}$ und $0.62 \mathrm{ml}$ Ether in $40 \mathrm{ml} \mathrm{CH}_{2} \mathrm{Cl}_{2}$

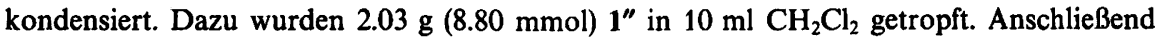
wurde $3.1 \mathrm{~d}$ bei $-78^{\circ} \mathrm{C}$ belassen, die orangerote Lösung dann auf konz. wäßr. Ammoniak gegossen und durchgeschüttelt. Nach nochmaligem Waschen mit Ammoniak-Lösung wurde die organische Phase über $\mathrm{CaCl}_{2}$ getrocknet und anschließend das Lösungsmittel abgezogen. Destillation des Rückstandes bei $100-110^{\circ} \mathrm{C} / 10 \mathrm{~Pa}$ lieferte $1.96 \mathrm{~g}$ eines 0.3:0.4:1-Gemisches von 8a", 10a" und 3a". Durch präp. Mitteldruckchromatographie (Kieselgel $15-25 \mu \mathrm{m}$, Petrolether Sdp. $\left.40-50^{\circ} \mathrm{C}, 12.5 \mathrm{ml} / \mathrm{min}, 7 \mathrm{bar}\right)$ konnten 8a" $(10 \%)\left(t_{\mathrm{R}}=12.5\right.$ $\min$ ), $10 \mathrm{a}^{\prime \prime}(14 \%)$ (zwei Stereoisomere, $c-10 \mathrm{a}^{\prime \prime}: t-10 \mathrm{a}^{\prime \prime} \approx 6^{39)}, t_{\mathrm{R}}=13.4 \mathrm{~min}$ ) und $3 \mathrm{a}^{\prime \prime}(34 \%)$ $\left(t_{\mathrm{R}}=16.7 \mathrm{~min}\right)$ voneinander getrennt werden.

3-Chlor-1,1-bis (4-methylphenyl)butan (3a"): ' $\mathrm{H}-\mathrm{NMR}\left(\mathrm{CCl}_{4}\right): \delta=1.43(\mathrm{~d}, J=6.7 \mathrm{~Hz}$; $3 \mathrm{H}, 4-\mathrm{H}$ ), $2.13-2.40$ und 2.23 (m und s; $8 \mathrm{H}, 2-\mathrm{H}, \mathrm{Ar}-\mathrm{CH}_{3}$ ), 3.67 (br. sext, $J \approx 6.6 \mathrm{~Hz} ; 1 \mathrm{H}$, 
3-H), 4.10 (br. t, $J=7.5 \mathrm{~Hz}$; $1 \mathrm{H}, 1-\mathrm{H}), 6.98$ (mc; $8 \mathrm{H}$, Aromaten-H). - 1,1-Bis(4-methylphenyl)-1-buten $\left(8 \mathrm{a}^{\prime \prime}\right):{ }^{1} \mathrm{H}-\mathrm{NMR}\left(\mathrm{CCl}_{4}\right): \delta=1.02(\mathrm{t}, J=7.6 \mathrm{~Hz} ; 3 \mathrm{H}, 4-\mathrm{H}), 2.10\left(\mathrm{dq}, J_{3,4}=\right.$ 7.6, $\left.J_{2,3}=7.1 \mathrm{~Hz} ; 2 \mathrm{H}, 3-\mathrm{H}\right), 2.28$ u. $2.35\left(2 \mathrm{~s} ; 6 \mathrm{H}, \mathrm{Ar}-\mathrm{CH}_{3}\right), 5.88(\mathrm{t}, J=7.1 \mathrm{~Hz} ; 1 \mathrm{H} ; 2-\mathrm{H})$, $6.80-7.10\left(\mathrm{~m} ; 8 \mathrm{H}\right.$, Aromaten-H). - trans-1,6-Dimethyl-3-(4-methylphenyl)indan $\left(t-10 \mathrm{a}^{\prime \prime}\right)$ : ${ }^{1} \mathrm{H}-\mathrm{NMR}\left(\mathrm{CDCl}_{3}\right): \delta=1.27\left(\mathrm{~d}, J=6.9 \mathrm{~Hz} ; 1-\mathrm{CH}_{3}\right), 1.38-1.75(\mathrm{~m} ; 2-\mathrm{H}), 2.30$ und $2.33(2 \mathrm{~s}$; Ar- $\mathrm{CH}_{3}$ ), 2.50-2.75 (m; 2-H), 3.30 (mc; 1-H), 4.36 (br. t, $\left.J \approx 7 \mathrm{~Hz} ; 3-\mathrm{H}\right), 6.75-7.18$ (m; Aromaten-H). $-{ }^{13} \mathrm{C}-\mathrm{NMR}\left(\mathrm{CDCl}_{3}\right): \delta=18.3\left(\mathrm{q} ; 1-\mathrm{CH}_{3}\right), 20.4$ und $21.0\left(2 \mathrm{q} ; \mathrm{Ar}-\mathrm{CH}_{3}\right), 45.0$ (t; C-2), 48.8 (d; C-1), 53.3 (d; C-3) und weitere Signale im Aromatenbereich. - cis-10a": ${ }^{1} \mathrm{H}-\mathrm{NMR}\left(\mathrm{CDCl}_{3}\right): \delta=1.34\left(\mathrm{~d}, J=6.79 \mathrm{~Hz} ; 3 \mathrm{H}, 1-\mathrm{CH}_{3}\right), 1.60(\mathrm{mc} ; 1 \mathrm{H}, 2-\mathrm{H}), 2.33$ und 2.35 (2 s; 6H, Ar- $\left.\mathrm{CH}_{3}\right), 2.68$ (mc; $\left.1 \mathrm{H}, 2-\mathrm{H}^{\prime}\right), 3.13(\mathrm{~m} ; 1 \mathrm{H}, 1-\mathrm{H}), 4.15\left(\mathrm{dd}, J_{1,2}=7.56 ; J_{1,2^{\circ}}=\right.$ $10.7 \mathrm{~Hz} ; 1 \mathrm{H}, 3-\mathrm{H}$ ), 6.77 und 6.94 (AB-System, $J=7.8 \mathrm{~Hz} ; 2 \mathrm{H}, 4-, 5-\mathrm{H}), 7.03-7.18$ (m; 5H, Aromaten-H). $-{ }^{13} \mathrm{C}-\mathrm{NMR}\left(\mathrm{CDCl}_{3}\right): \delta=19.1$ (q; 1- $\left.\mathrm{CH}_{3}\right), 21.0$ und 21.4 (2 q; Ar-CH $\left.{ }_{3}\right), 38.2$ (d; C-1), 46.9 (t; C-2), 49.9 (d; C-3), 123.5, 124.3, 127.1, 128.2, 129.1 (5 d, C-4, -5, -7, 3-Phenyl$\mathrm{C}_{o}, \mathrm{C}_{m}$ ), 135.7, 136.1, 142.0, 144.2, 148.9 (5 s; C-6, -8; -9, 3-Phenyl- $\mathrm{C}_{p}, \mathrm{C}_{i}$ ).

3. Isobuten (4d) und Bis(4-methylphenyl)methylchlorid (1"): $60 \mathrm{ml}$ absol. $\mathrm{CH}_{2} \mathrm{Cl}_{2}$ wurden auf $-78^{\circ} \mathrm{C}$ gekühlt, $1.80 \mathrm{~g}(32.1 \mathrm{mmol}) 4 \mathrm{~d}$ einkondensiert und mit einer Lösung von $0.259 \mathrm{~g}$


von $2.10 \mathrm{~g}(9.10 \mathrm{mmol}) \mathbf{1}^{\prime \prime}$ in $20 \mathrm{ml} \mathrm{CH} \mathrm{Cl}_{2}$ innerhalb von 30 min getropft. Nach weiteren 30 min wurde auf konz. wäßr. Ammoniak gegossen und wie oben aufgearbeitet. Destillation $\left(120-130^{\circ} \mathrm{C}(\mathrm{Bad}) / 0.13 \mathrm{~Pa}\right)$ ergab $2.49 \mathrm{~g}(95 \%)$ 3-Chlor-3-methyl-1,1-bis(4-methylphenyl)butan (3d") als farblose Flüssigkeit, die beim Aufbewahren im Kühlschrank zu einer farblosen Masse erstarrte. - ${ }^{1} \mathrm{H}-\mathrm{NMR}\left(\mathrm{CCl}_{4}\right): \delta=1.37\left(\mathrm{~s} ; 6 \mathrm{H},\left(\mathrm{CH}_{3}\right)_{2} \mathrm{C}\right), 2.23\left(\mathrm{~s} ; 6 \mathrm{H}, \mathrm{Ar}-\mathrm{CH}_{3}\right), 2.53$ (d, $J=6 \mathrm{~Hz} ; 2 \mathrm{H}, 2-\mathrm{H}), 4.27(\mathrm{t}, J=6 \mathrm{~Hz} ; 1 \mathrm{H}, 1-\mathrm{H}), 6.80-7.23$ (m; 8H, Aromaten-H). MS (70 eV): $m / z=288,286\left(1.2 \%, 3.5 \%, \mathrm{M}^{+}\right), 195(100), 180(15), 166.2\left(m^{*}, 195 \rightarrow 180\right)$, $165(19), 151.3\left(m^{*}, 180 \rightarrow 165\right)$.

\section{$\mathrm{C}_{19} \mathrm{H}_{23} \mathrm{Cl}(286.9) \quad$ Ber. C $79.56 \mathrm{H} 8.08$ Gef. C 79.39 H 8.17}

4. 2-Methyl-1-buten (4e) und Diphenylmethylchlorid (1): $\mathrm{Zu}$ einer Lösung von $2.2 \mathrm{~g}$ (16 mmol) $\mathrm{ZnCl}_{2}$ in $2.7 \mathrm{ml}$ Ether und $35 \mathrm{ml} \mathrm{CH}_{2} \mathrm{Cl}_{2}$ wurden bei $-78^{\circ} \mathrm{C} 4.0 \mathrm{~g}(20 \mathrm{mmol}) \mathrm{1}^{\prime}$ in $10 \mathrm{ml} \mathrm{CH} \mathrm{Cl}_{2}$ gegeben und anschließend innerhalb von $1 \mathrm{~h} 2.8 \mathrm{~g}(40 \mathrm{mmol}) 4 \mathrm{e}$ in $10 \mathrm{ml}$ $\mathrm{CH}_{2} \mathrm{Cl}_{2}$ zugetropft. Nach $1 \mathrm{~h}$ wurde die gelbe Lösung wie oben aufgearbeitet, wobei man $5.1 \mathrm{~g}$ einer blaßgelben Flüssigkeit erhielt, deren Destillation $4.8 \mathrm{~g}(89 \%)$ 3-Chlor-3-methyl1,1-diphenylpentan (3e') als farbloses Öl mit Sdp. $100-112^{\circ} \mathrm{C}(\mathrm{Bad}) / 0.1 \mathrm{~Pa}$ lieferte. $-{ }^{1} \mathrm{H}-$ NMR $\left(\mathrm{CCl}_{4}\right): \delta=0.92$ (verzerrtes t; $\left.3 \mathrm{H}, 5-\mathrm{H}\right), 1.27$ (s; 3 H, 3- $\mathrm{CH}_{3}$ ), 1.7 (mc; $\left.2 \mathrm{H}, 4-\mathrm{H}\right), 2.58$ $(\mathrm{d}, J=6.0 \mathrm{~Hz} ; 2 \mathrm{H}, 2-\mathrm{H}), 4.33$ (t, $J=6.0 \mathrm{~Hz} ; 1 \mathrm{H}, 1-\mathrm{H}), 7.20$ (mc; $10 \mathrm{H}$, Aromaten-H). MS (70 eV): $m / z=236\left(\mathrm{M}^{+}-\mathrm{HCl}, 4 \%\right), 207$ (13), 168 (15), 167 (100), 165 (25), 152 (17).

\section{$\mathrm{C}_{18} \mathrm{H}_{21} \mathrm{Cl}(272.8)$ Ber. C 79.24 H 7.76 Gef. C 78.90 H 8.15}

5. 2-Methyl-1-buten (4e) und Bis(4-methylphenyl)methylchlorid (1"): Eine Lösung von $1.10 \mathrm{~g}(15.7 \mathrm{mmol}) 4 \mathrm{e}$ in $50 \mathrm{ml} \mathrm{CH}_{2} \mathrm{Cl}_{2}$ wurde auf $-78^{\circ} \mathrm{C}$ gekühlt und mit einer Lösung von $0.556 \mathrm{~g}$ (4.08 mmol) $\mathrm{ZnCl}_{2}$ in $0.66 \mathrm{ml}$ Ether und $1.33 \mathrm{ml} \mathrm{CH} \mathrm{Cl}_{2}$ versetzt. Dann wurden innerhalb von $15 \mathrm{~min} 3.00 \mathrm{~g}(13.0 \mathrm{mmol}) \mathrm{1}^{\prime \prime}$ in $10 \mathrm{ml} \mathrm{CH} \mathrm{Cl}_{2}$ zugetropft. Nach $3 \mathrm{~h}$ wurde die gelbe Lösung wie oben aufgearbeitet. Vakuumdestillation ergab $3.57 \mathrm{~g} \mathrm{(91 \% )} 3$-Chlor3-methyl-1,1-bis(4-methylphenyl)pentan (3e') als farblose Flüssigkeit mit Sdp. $110-120^{\circ} \mathrm{C}$ (Bad)/0.13 Pa. $-{ }^{1} \mathrm{H}-\mathrm{NMR}\left(\mathrm{CCl}_{4}\right): \delta=0.90$ (verzerrtes t, $\left.J=7 \mathrm{~Hz} ; 3 \mathrm{H}, 5-\mathrm{H}\right), 1.27(\mathrm{~s} ; 3 \mathrm{H}$, 3- $\left.\mathrm{CH}_{3}\right), 1.58(\mathrm{mc} ; 2 \mathrm{H}, 4-\mathrm{H}), 2.23\left(\mathrm{~s} ; 6 \mathrm{H}, \mathrm{Ar}-\mathrm{CH}_{3}\right) ; 2.53(\mathrm{~d}, J=6 \mathrm{~Hz} ; 2 \mathrm{H}, 2-\mathrm{H}), 4.23$ (t, $J=$ $6 \mathrm{~Hz} ; 1 \mathrm{H}, 1-\mathrm{H}), 6.80-7.20$ (m; $8 \mathrm{H}$, Aromaten-H).

$$
\mathrm{C}_{20} \mathrm{H}_{25} \mathrm{Cl}(300.9) \quad \text { Ber. C } 79.84 \text { H } 8.38 \text { Gef. C } 80.05 \text { H } 8.14
$$

6. 2-Methyl-1-penten (4f) und Bis (4-methylphenyl)methylchlorid (1'): $\mathrm{Zu} 1.60 \mathrm{~g}(19.0 \mathrm{mmol})$ $4 \mathrm{f}$ in Gegenwart von $0.52 \mathrm{~g}$ (3.8 mmol) $\mathrm{ZnCl}_{2}$ und $0.62 \mathrm{ml}$ Diethylether in $50 \mathrm{ml} \mathrm{CH}_{2} \mathrm{Cl}_{2}$

Chem. Ber. 119 (1986) 
tropfte man bei $-78^{\circ} \mathrm{C} 3.00 \mathrm{~g}(13.0 \mathrm{mmol}) 1^{\prime \prime}$ in $20 \mathrm{ml} \mathrm{CH} \mathrm{Cl}_{2}$. Nach 30 min wurde die gelbe Lösung wie oben aufgearbeitet und lieferte $3.73 \mathrm{~g}(91 \%) 3$-Chlor-3-methyl-1,1-bis (4methylphenyl)hexan (3f') als farblose, viskose Flüssigkeit mit Sdp. $125-130^{\circ} \mathrm{C}$ (Bad)/ $0.5 \mathrm{~Pa}$. - ${ }^{1} \mathrm{H}-\mathrm{NMR}\left(\mathrm{CCl}_{4}\right): \delta=0.6-1.0(\mathrm{~m} ; 3 \mathrm{H}, 6-\mathrm{H}), 1.27\left(\mathrm{~s} ; 3 \mathrm{H}, 3-\mathrm{CH}_{3}\right), 1.47-1.70(\mathrm{~m}$; $4 \mathrm{H}, 4-, 5-\mathrm{H}), 2.27\left(\mathrm{~s} ; 6 \mathrm{H}, \mathrm{Ar}-\mathrm{CH}_{3}\right), 2.52(\mathrm{~d}, J=6.0 \mathrm{~Hz} ; 2 \mathrm{H}, 2-\mathrm{H}), 4.22(\mathrm{t}, J=6.0 \mathrm{~Hz} ; 1 \mathrm{H}$, 1-H), 7.05 (mc; $8 \mathrm{H}$, Aromaten-H).

\section{$\mathrm{C}_{21} \mathrm{H}_{27} \mathrm{Cl}$ (314.9) Ber. C 80.10 H 8.64 Gef. C 80.18 H 8.55}

7. 2-Methyl-2-buten (4g) und Diphenylmethylchlorid (1'): Zu $3.01 \mathrm{~g}(42.9 \mathrm{mmol}) 4 \mathrm{~g}$ in $30 \mathrm{ml}$ $\mathrm{CH}_{2} \mathrm{Cl}_{2}$ wurden bei $-78^{\circ} \mathrm{C}$ zuerst eine Lösung von $0.80 \mathrm{~g}(5.9 \mathrm{mmol}) \mathrm{ZnCl}_{2}$ in $1.0 \mathrm{ml} \mathrm{Di}$ ethylether und $2.0 \mathrm{ml} \mathrm{CH}{ }_{2} \mathrm{Cl}_{2}$ und dann innerhalb von $15 \min 4.29 \mathrm{~g}(21.2 \mathrm{mmol}) \mathbf{1}^{\prime}$ in $10 \mathrm{ml}$ $\mathrm{CH}_{2} \mathrm{Cl}_{2}$ getropft. Nach $3 \mathrm{~h}$ wurde wie oben aufgearbeitet, wobei $5.41 \mathrm{~g}$ eines Rückstands erhalten wurden, der erstarrte. Umkristallisation aus $n$-Pentan lieferte 5.12 g (89\%) 3-Chlor2,3-dimethyl-1,1-diphenylbutan (3g') als farblose Rhomboeder mit Schmp. 56-57 ${ }^{\circ} \mathrm{C} .-{ }^{1} \mathrm{H}$ NMR $\left(\mathrm{CCl}_{4}\right): \delta=0.97\left(\mathrm{~d}, J=7.3 \mathrm{~Hz} ; 3 \mathrm{H}, 2-\mathrm{CH}_{3}\right), 1.35(\mathrm{~s} ; 3 \mathrm{H}, 4-\mathrm{H}), 1.48\left(\mathrm{~s} ; 3 \mathrm{H}, 3-\mathrm{CH}_{3}\right)$, 2.73 (quint, $J=7.1 \mathrm{~Hz} ; 1 \mathrm{H}, 2-\mathrm{H}), 4.27$ (d, $J=7.3 \mathrm{~Hz} ; 1 \mathrm{H}, 1-\mathrm{H}), 7.00-7.33(\mathrm{~m} ; 10 \mathrm{H}$, Aromaten-H). - MS (96 eV): $m / z=274,272\left(0.9 \%, 3 \%, M^{+}\right), 168(42), 167(100), 166(13)$, 165 (33).

$$
\mathrm{C}_{18} \mathrm{H}_{21} \mathrm{Cl} \text { (272.8) Ber. C } 79.24 \mathrm{H} 7.76 \text { Gef. C } 79.28 \text { H } 7.70
$$

Versuch mit höherer Konzentration an $\mathrm{ZnCl}_{2} \cdot \mathrm{Et}_{2} \mathrm{O}$ : Man versetzt $2.7 \mathrm{~g}(39 \mathrm{mmol}) 4 \mathrm{~g}$ in $30 \mathrm{ml} \mathrm{CH} \mathrm{Cl}_{2}$ bei $-78^{\circ} \mathrm{C}$ mit einer Lösung von $\left.2.2 \mathrm{~g} \mathrm{(16} \mathrm{mmol}\right) \mathrm{ZnCl}_{2}$ in $2.7 \mathrm{ml}$ Ether und $15.3 \mathrm{ml} \mathrm{CH} \mathrm{Cl}_{2}$ und tropft anschließend innerhalb von $20 \mathrm{~min} 3.5 \mathrm{~g}(17 \mathrm{mmol}) \mathbf{1}^{\prime}$ in $10 \mathrm{ml}$ $\mathrm{CH}_{2} \mathrm{Cl}_{2}$ zu. Gießt man nach $70 \mathrm{~min}$ auf konz. wäßr. Ammoniak, erhält man $4.3 \mathrm{~g}$ einer farblosen Flüssigkeit, die $\mathbf{3} \mathbf{g}^{\prime}$ und $\mathbf{9} \mathbf{g}^{\prime}$ (3:1) sowie eine Spur $10 \mathbf{g}^{\prime}$ enthält.

8. 2-Methyl-2-buten (4g) und Bis(4-methylphenyl)methylchlorid (1"): $\mathrm{Zu}$ einer Lösung von

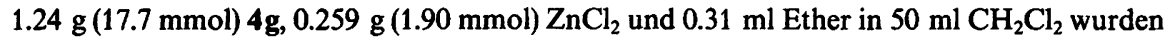
bei $-78^{\circ} \mathrm{C}$ innerhalb von $10 \mathrm{~min} 2.08 \mathrm{~g}(9.01 \mathrm{mmol}) \mathbf{1}^{\prime \prime}$ in $20 \mathrm{ml} \mathrm{CH}_{2} \mathrm{Cl}_{2}$ getropft. $\mathrm{Nach}$ 30 min wurde die gelbe Lösung auf konz. wäßr. Ammoniak gegossen und wie oben aufgearbeitet. Man erhielt eine viskose, farblose Masse, die nach dem ${ }^{1} H-N M R-S p e k t r u m$ aus 3-Chlor-2,3-dimethyl-1,1-bis(4-methylphenyl)butan (3 $\left.\mathrm{g}^{\prime \prime}\right)$ und 1,1,2,6-Tetramethyl-3-(4-methylphenyl)indan $\left(\mathbf{1 0}^{\prime \prime}\right.$ ) im Verhältnis $90: 10$ bestand. Umkristallisation aus Petrolether (Sdp. $\left.40-60^{\circ} \mathrm{C}\right)$ ergab $1.85 \mathrm{~g}(68 \%) 3 \mathrm{~g}$ " in farblosen Säulen mit Schmp. $62-64^{\circ} \mathrm{C}$. - 'H-NMR $\left(\mathrm{CCl}_{4}\right): \delta=0.93\left(\mathrm{~d}, J=7.2 \mathrm{~Hz} ; 3 \mathrm{H}, 2-\mathrm{CH}_{3}\right), 1.33(\mathrm{~s} ; 3 \mathrm{H}, 4-\mathrm{H}), 1.47\left(\mathrm{~s} ; 3 \mathrm{H}, 3-\mathrm{CH}_{3}\right), 2.27$ (s; $6 \mathrm{H}, \mathrm{Ar}-\mathrm{CH}_{3}$ ), 2.68 (quint, $J=7.2 \mathrm{~Hz} ; 1 \mathrm{H}, 2-\mathrm{H}$ ), 4.15 (d, $\left.J=7.2 \mathrm{~Hz} ; 1 \mathrm{H}, 1-\mathrm{H}\right), 6.83-7.30$ (mc; 8 H, Aromaten-H). - MS (70 eV): $m / z=302,300\left(1.4 \%, 4.1 \%, \mathrm{M}^{+}\right), 249(4), 195(100)$, 180 (8.6), 165 (11).

9. 2,3-Dimethyl-2-buten (4h) und Diphenylmethylchlorid (1'): $\mathrm{Zu}$ einer Lösung von $3.60 \mathrm{~g}$ (42.8 mmol) $4 \mathrm{~h}, 1.95 \mathrm{~g}$ (14.3 mmol) $\mathrm{ZnCl}_{2}$ und $2.3 \mathrm{ml}$ Diethylether in $35 \mathrm{ml} \mathrm{CH}_{2} \mathrm{Cl}_{2}$ wurden bei $-78^{\circ} \mathrm{C}$ innerhalb von $20 \mathrm{~min} 4.07 \mathrm{~g}(20.1 \mathrm{mmol}) \mathrm{1}^{\prime}$ in $10 \mathrm{ml} \mathrm{CH}_{2} \mathrm{Cl}_{2}$ getropft. $\mathrm{Nach} 2 \mathrm{~h}$ 40 min wurde die Reaktion durch Zusatz von wäßr. Ammoniak abgebrochen. Arbeitete man in der oben beschriebenen Weise auf, erhielt man $5.24 \mathrm{~g}$ einer blaßgelben Flüssigkeit, die nach ${ }^{1} \mathrm{H}-\mathrm{NMR}$-Spektrum aus 1,1,2,2-Tetramethyl-3-phenylindan $\left(\mathbf{1 0 h}^{\prime}\right)$ und 3-Chlor-2,2,3trimethyl-1,1-diphenylbutan (3h') im Verhältnis $65: 35$ bestand. Destillation bei $90-95^{\circ} \mathrm{C}$ (Bad)/0.1 Pa lieferte $4.95 \mathrm{~g} \mathrm{(93 \% )} \mathrm{einer} \mathrm{farblosen} \mathrm{Flüssigkeit} \mathrm{gleicher} \mathrm{Zusammensetzung.}$ Verwendet man nur $40 \%$ der oben eingesetzten $\mathrm{ZnCl}_{2}$ /Ether-Menge und bricht die Umsetzung nach $1 \mathrm{~h} \mathrm{ab}$, bevor $\mathbf{1}^{\prime}$ vollständig verbraucht ist, erhält man $10 \mathrm{~h}^{\prime}$ und $3 \mathrm{~h}^{\prime}$ im Verhältnis 44:66. An diesem Gemisch wurden die spektroskopischen Daten von $3 \mathbf{h}^{\prime}$ bestimmt: ${ }^{1} \mathbf{H}-$ $\operatorname{NMR}\left(\mathrm{CCl}_{4}\right): \delta=1.25\left(\mathrm{~s} ; 6 \mathrm{H}, 2-\mathrm{CH}_{3}\right), 1.40\left(\mathrm{~s} ; 6 \mathrm{H},\left(\mathrm{CH}_{3}\right)_{2} \mathrm{CCl}\right), 4.35(\mathrm{~s} ; 1 \mathrm{H}, 1-\mathrm{H})$, Aromaten$\mathrm{H}$ sind durch die Signale von $10 h^{\prime}$ überlagert. 
1,1,2,2-Tetramethyl-3-phenylindan (10 h') wurde mit $93 \%$ Ausbeute erhalten, als das bei $-78^{\circ} \mathrm{C}$ aus $4 \mathrm{~h}$ und $1^{\prime}$ gebildete Produktgemisch vor Auswaschen des Katalysators mit Ammoniak noch $34 \mathrm{~h}$ bei $0^{\circ} \mathrm{C}$ belassen wurde. Farblose Würfel mit Schmp. $62-63^{\circ} \mathrm{C}$ (aus Pentan). $-{ }^{1} \mathrm{H}-\mathrm{NMR}\left(\mathrm{CCl}_{4}\right): \delta=0.50\left(\mathrm{~s} ; 3 \mathrm{H}, 2-\mathrm{CH}_{3}\right), 1.03\left(\mathrm{~s} ; 3 \mathrm{H}, 2-\mathrm{CH}_{3}\right), 1.10(\mathrm{~s} ; 3 \mathrm{H}$, 1- $\left.\mathrm{CH}_{3}\right), 1.23$ (s; 3 H, 1- $\left.\mathrm{CH}_{3}\right), 4.20(\mathrm{~s} ; 1 \mathrm{H}, 3-\mathrm{H}), 6.95-7.20\left(\mathrm{~m} ; 9 \mathrm{H}\right.$, Aromaten-H). $-{ }^{13} \mathrm{C}-$ $\operatorname{NMR}\left(\mathrm{CDCl}_{3}\right): \delta=20.78,20.99,21.57,26.09\left(4 \mathrm{q} ; \mathrm{CH}_{3}\right), 47.51,50.24$ (2 s; C-1, -2), 59.21 (d; C-3), 122.01, 124.80, 125.98, 125.35, 126.80 (5 d; 5 Aromaten-C), 127.59, 130.20 (2 d; Phenyl$\left.\mathrm{C}_{o}, \mathrm{C}_{m}\right), 139.27,142.58,152.46$ (3 s; Aromaten-C). - UV ( $n$-Pentan): $\lambda_{\max }(\varepsilon)=272.5 \mathrm{~nm}$ (1100), 265.8 (1200), 259.2 (1000). - MS (96 eV): $m / z=250\left(\mathrm{M}^{+}, 78 \%\right), 235(100), 207$ (27), 193 (49), 157 (23), 105 (26).

\section{$\mathrm{C}_{19} \mathrm{H}_{22}$ (250.4) Ber. C 91.14 H 8.86 Gef. C 91.53 H 8.33}

10. Methylencyclobutan (4i) und Bis(4-methylphenyl)methylchlorid (1"): Eine Lösung von

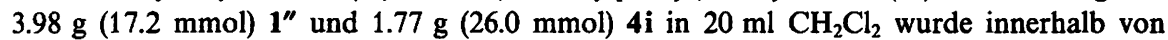
$25 \mathrm{~min} \mathrm{zu}$ einer auf $-78^{\circ} \mathrm{C}$ gekühlten Lösung von $278 \mathrm{mg}(2.04 \mathrm{mmol}) \mathrm{ZnCl}_{2}$ in $0.33 \mathrm{ml}$ Ether und $67 \mathrm{ml} \mathrm{CH} \mathrm{Cl}_{2}$ getropft. Nach $30 \mathrm{~min}$ wurde, wie oben beschrieben, aufgearbeitet. Man erhielt eine farblose trübe Flüssigkeit, die in Petrolether $\left(\mathrm{Sdp} .40-50^{\circ} \mathrm{C}\right.$ ) aufgenommen, über Kieselgel 60 filtriert und anschließend aus Ethanol umkristallisiert wurde: $4.49 \mathrm{~g}(87 \%)$ 1-[2,2-Bis (4-methylphenyl)ethyl]-1-chlorcyclobutan (3i") in farblosen gestreckten Prismen mit Schmp. $40-41^{\circ} \mathrm{C} .-{ }^{1} \mathrm{H}-\mathrm{NMR}\left(\mathrm{CDCl}_{3}\right): \delta=1.50-1.70(\mathrm{~m} ; 1 \mathrm{H}$, Cyclobutan-3-H), 1.90-2.24 (m; 5H, Cyclobutan-2,3,4-H), 2.28 (s; 6H, Ar- $\mathrm{CH}_{3}$ ), 2.59 (br. d, $J=7.0 \mathrm{~Hz} ; 2 \mathrm{H}$, $\mathrm{CH}_{2} \mathrm{CH}$ ), 4.35 (t, $\left.J=7.0 \mathrm{~Hz} ; 1 \mathrm{H}, \mathrm{CHAr}_{2}\right), 7.07,7.17\left(\mathrm{AA}^{\prime} \mathrm{BB}^{\prime}-\right.$ System, $J=7.9 \mathrm{~Hz} ; 8 \mathrm{H}$, Aromaten-H). $-{ }^{13} \mathrm{C}-\mathrm{NMR}\left(\mathrm{CDCl}_{3}\right): \delta=16.11$ (t; Ring-C-3), 20.96 (q; $\left.\mathrm{CH}_{3}\right), 39.10$ (t; RingC-2,4), 47.14 (d; $C$ HAr ${ }_{2}$ ), 48.11 (t; Ethyl- $\mathrm{CH}_{2}$ ), 73.50 (s; Ring-C-1), 127.77, 129.04 (2 d; ArylCH), 135.50 (s; Aryl-C-4), 142.00 (s; Aryl-C-1).

\section{$\mathrm{C}_{20} \mathrm{H}_{23} \mathrm{Cl}$ (298.9) Ber. C 80.38 H 7.76 Gef. C 80.45 H 7.77}

11. Methylencyclopentan (4j) und Bis(4-methylphenyl)methylchlorid (1"): $\mathrm{Zu}$ einer Lösung von $522 \mathrm{mg}$ (6.36 mmol) 4 j, $278 \mathrm{mg}(2.04 \mathrm{mmol}) \mathrm{ZnCl}_{2}$ und $0.33 \mathrm{ml} \mathrm{Ether} \mathrm{in} 50 \mathrm{ml} \mathrm{CH}_{2} \mathrm{Cl}_{2}$ wurden bei $-78^{\circ} \mathrm{C}$ innerhalb von $30 \mathrm{~min} 1.03 \mathrm{~g}(4.46 \mathrm{mmol}) 1^{\prime \prime}$ in $20 \mathrm{ml} \mathrm{CH}_{2} \mathrm{Cl}_{2}$ getropft. Nach 15 min wurde die gelbe Lösung wie oben aufgearbeitet. Umkristallisation des Rohprodukts aus Petrolether (Sdp. $40-45^{\circ} \mathrm{C}$ ) lieferte $1.15 \mathrm{~g}(82 \%)$ 1-[2,2-Bis(4-methylphenyl)ethyl]-1-chlorcyclopentan (3j") in farblosen Nadeln mit Schmp. $40-42^{\circ} \mathrm{C}$. $-{ }^{1} \mathrm{H}-\mathrm{NMR}$ $\left(\mathrm{CCl}_{4}\right): \delta=1.10-2.00\left(\mathrm{~m} ; 8 \mathrm{H}\right.$, Ring-H), $2.27\left(\mathrm{~s} ; 6 \mathrm{H}, \mathrm{Ar}-\mathrm{CH}_{3}\right), 2.58(\mathrm{~d}, J=6.5 \mathrm{~Hz} ; 2 \mathrm{H}$, $\left.\mathrm{CH}_{2} \mathrm{CH}\right), 4.45\left(\mathrm{t}, \mathrm{J}=6.5 \mathrm{~Hz} ; 1 \mathrm{H}, \mathrm{CHAr}_{2}\right), 6.77-7.23$ (m; $8 \mathrm{H}$, Aromaten-H). - MS (70 eV): $m / z=314,312\left(1 \%, 3 \%, M^{+}\right), 196(17), 195(100), 180(10), 165(12), 166.2\left(m^{*}, 195 \rightarrow 180\right)$, $151.3\left(m^{*}, 180 \rightarrow 165\right)$.

Beim Umkristallisationsversuch aus Ethanol oder Filtrieren einer Petrolether-Lösung über Kieselgel 60 entsteht aus 3j" das $\mathrm{HCl}$-Eliminierungsprodukt 2-(1-Cyclopenten-1-yl)1,1-bis (4-methylphenyl)ethan als farblose Flüssigkeit. $\left({ }^{1} \mathrm{H}-\mathrm{NMR}\left(\mathrm{CCl}_{4}\right): \delta=1.43-2.33\right.$ (br. m; Ring-H), 2.27 (s; $\mathrm{Ar}-\mathrm{CH}_{3}$ ), 2.73 (br. d, $J \approx 8 \mathrm{~Hz} ; 1-\mathrm{H}$ ), 4.03 (t, $J \approx 8 \mathrm{~Hz}$; $\mathrm{CHAr}_{2}$ ), 5.17 (br. s; Vinyl-H), 7.00 (mc; Aromaten-H).)

12. Methylencyclohexan (4k) und Bis(4-methylphenyl)methylchlorid (1"): $\mathrm{Zu}$ einer auf $-78^{\circ} \mathrm{C}$ gekühlten Lösung von $2.61 \mathrm{~g}(27.1 \mathrm{mmol}) 4 \mathrm{k}$ in $50 \mathrm{ml} \mathrm{CH} \mathrm{Cl}_{2}$ wurden $278 \mathrm{mg}(2.04$ mmol) $\mathrm{ZnCl}_{2}$ in $0.33 \mathrm{ml}$ Ether und $0.67 \mathrm{ml} \mathrm{CH}_{2} \mathrm{Cl}_{2}$ gegeben; dann wurden innerhalb von $15 \mathrm{~min} 2.44 \mathrm{~g}$ (10.6 mmol) $1^{\prime \prime}$ in $30 \mathrm{ml} \mathrm{CH}_{2} \mathrm{Cl}_{2}$ zugetropft. Nach $1 \mathrm{~h}$ wurde die intensiv gelbe Lösung in gewohnter Weise aufgearbeitet. Da beim Versuch der Vakuumdestillation (1 Pa) Zersetzung erfolgte, wurde das ölige Rohprodukt in Petrolether (Sdp. $40-60^{\circ} \mathrm{C}$ ) gelöst und über eine $10 \times 1.5$-cm-Säule mit Kieselgel 60 filtriert. Nach Abziehen des Lö- 
sungsmittels wurden $3.00 \mathrm{~g}(87 \%)$ analysenreines 1 -[2,2-Bis(4-methylphenyl)ethyl]-1-chlorcyclohexan (3k") als farblose viskose Flüssigkeit erhalten. - ${ }^{1} \mathrm{H}-\mathrm{NMR}\left(\mathrm{CCl}_{4}\right): \delta=$ 0.83-2.00 (m; 10H, Ring-H), 2.25 (s; 6 H, Ar- $\mathrm{CH}_{3}$ ), 2.50 (d, $\mathrm{J}=6.0 \mathrm{~Hz} ; 2 \mathrm{H}, \mathrm{CH}_{2} \mathrm{CH}$ ), 4.40 (t, $\left.J=6.0 \mathrm{~Hz} ; 1 \mathrm{H}, \mathrm{CHAr}_{2}\right), 6.77-7.23(\mathrm{~m} ; 8 \mathrm{H}$, Aromaten-H). $-\mathrm{MS}(70 \mathrm{eV}): \mathrm{m} / z=328$, $326\left(0.02 \%, 0.07 \%, \mathrm{M}^{+}\right), 290(21), 234(11), 208(13), 198(26), 195(100), 180(11), 166.2\left(m^{*}\right.$, $195 \rightarrow 180$ ).

\section{$\mathrm{C}_{22} \mathrm{H}_{27} \mathrm{Cl}$ (326.9) Ber. C 80.83 H 8.33 Gef. C 80.98 H 8.41}

13. 1-Methyl-1-cyclohexen (4I) und Bis(4-methylphenyl)methylchlorid (1"): $\mathrm{Zu}$ einer auf $-78^{\circ} \mathrm{C}$ gekühlten Lösung von $1.12 \mathrm{~g}(11.6 \mathrm{mmol}) 41,278 \mathrm{mg}(2.04 \mathrm{mmol}) \mathrm{ZnCl}_{2}$ und $0.33 \mathrm{ml}$ Ether in $40 \mathrm{ml} \mathrm{CH} \mathrm{Cl}_{2}$ wurden innerhalb von $15 \mathrm{~min} 2.00 \mathrm{~g}(8.67 \mathrm{mmol}) \mathrm{1}^{\prime \prime}$ in $10 \mathrm{ml} \mathrm{CH} \mathrm{Cl}_{2}$ getropft. Nach $7 \mathrm{~h}$ wurde die braungelbe Lösung auf konz. wäßr. Ammoniak gegossen und wie oben aufgearbeitet. Das dabei erhaltene Öl besteht nach ${ }^{1} \mathrm{H}-\mathrm{NMR}$-Analyse aus $3 \mathrm{I}^{\prime \prime}$ und Spuren einer Verbindung mit ${ }^{1} \mathrm{H}$-NMR-Signalen $\left(\mathrm{CCl}_{4}\right)$ bei $\delta=4.15$ (d) und 1.10 (s), die mit dem Indan $101^{\prime \prime}$ vereinbar wären. Umkristallisation aus Petrolether (Sdp. $\left.40-50^{\circ} \mathrm{C}\right)$ ergab $2.39 \mathrm{~g}(84 \%)$ 2-[Bis (4-methylphenyl) methyl]-1-chlor-1-methylcyclohexan (31") in garbenförmigen farblosen Kristallaggregaten mit Schmp. $90-91^{\circ} \mathrm{C}$. Die Konfiguration von 31 ist unbekannt. - ${ }^{1} \mathrm{H}-\mathrm{NMR}\left(\mathrm{CCl}_{4}\right): \delta=1.10-2.27$ (m; $8 \mathrm{H}$, Ring-H), 1.30 (s; 3H, 1-CH ${ }_{3}$ ), 2.28 (s; 6H, Ar- $\mathrm{CH}_{3}$ ), 2.67 (mc; $\left.1 \mathrm{H}, 2-\mathrm{H}\right), 4.47$ (d, $\left.J \approx 6 \mathrm{~Hz} ; 1 \mathrm{H}, \mathrm{Ar}_{2} \mathrm{CH}\right), 6.87-7.27$ (m; Aromaten-H).

$$
\mathrm{C}_{22} \mathrm{H}_{27} \mathrm{Cl} \text { (326.9) Ber. C } 80.83 \text { H } 8.33 \text { Gef. C } 80.93 \text { H } 8.05
$$

\section{Irreversibilität der Additionsreaktionen}

1. Kinetische Stabilität der Additionsprodukte 3: $\mathrm{Zu}$ einer Lösung von $0.726 \mathrm{~g}(2.81 \mathrm{mmol})$ 3d', $0.30 \mathrm{~g}(2.0 \mathrm{mmol}) \mathrm{ZnCl}_{2}$ und $0.3 \mathrm{ml}$ Ether in $10 \mathrm{ml} \mathrm{CH} \mathrm{Cl}_{2}$ wurden bei $-78^{\circ} \mathrm{C} 1.000 \mathrm{~g}$ (14.3 mmol) $4 \mathrm{~g}$ in $10 \mathrm{ml} \mathrm{CH}_{2} \mathrm{Cl}_{2}$ getropft. Nach $6 \mathrm{~h}$ wurde auf konz. wäßr. Ammoniak gegossen. Nach Abziehen des Lösungsmittels blieben $0.70 \mathrm{~g}$ einer farblosen Flüssigkeit zurück, die nach dem ${ }^{1} \mathrm{H}-\mathrm{NMR}$-Spektrum aus unverändertem $3 \mathbf{d}^{\prime}$ bestand.

In gleicher Weise wurden $0.169 \mathrm{~g}(0.620 \mathrm{mmol}) 3 \mathrm{~g}^{\prime} 5 \mathrm{~h}$ mit $0.500 \mathrm{~g} 4 \mathrm{~d}$ in Gegenwart von $0.30 \mathrm{~g} \mathrm{ZnCl}_{2}$ und $0.3 \mathrm{ml}$ Ether behandelt, ohne daß eine Veränderung eintrat.

\section{Kreuzungsexperimente}

a) Darstellung der Alkene $4 \mathrm{n}$ und $4 \mathrm{o}: 12.0 \mathrm{~g}(46.4 \mathrm{mmol}) 3 \mathrm{~d}^{\prime}$ wurden in $50 \mathrm{ml}$ tert-Butylalkohol gelöst, mit $19.8 \mathrm{~g}(176 \mathrm{mmol}) \mathrm{Kalium}$-tert-butoxid versetzt und $5 \mathrm{~h}$ auf $110^{\circ} \mathrm{C}$ erhitzt. Die Mischung wurde auf Wasser gegossen, mit Dichlormethan extrahiert, der Extrakt über $\mathrm{CaCl}_{2}$ getrocknet und das Lösungsmittel abgezogen, wobei man $9.4 \mathrm{~g}$ Rückstand erhielt. Destillation bei $85-90^{\circ} \mathrm{C}(\mathrm{Bad}) / 0.1 \mathrm{~Pa}$ ergab $9.14 \mathrm{~g}(89 \%)$ 2-Methyl-4,4-diphenyl-1buten (4n): ${ }^{1} \mathrm{H}-\mathrm{NMR}\left(\mathrm{CCl}_{4}\right): \delta=1.67$ (br. s; $\left.3 \mathrm{H}, 2-\mathrm{CH}_{3}\right), 2.73$ (br. d, $J=8.0 \mathrm{~Hz} ; 2 \mathrm{H}, 3-\mathrm{H}$ ), $4.15(\mathrm{t}, J=8.0 \mathrm{~Hz}$; $1 \mathrm{H}, 4-\mathrm{H}), 4.67$ (mc; $2 \mathrm{H}, 1-\mathrm{H}), 7.20$ (s; Aromaten-H). - IR (Film): $v=$ 3060, $3020 \mathrm{~cm}^{-1}$ (Aromaten-CH), 2960, 2920, 2880, $2840(\mathrm{CH}), 1640(\mathrm{C}=\mathrm{C}), 1590,1580$, 1485 (Aromaten-Gerüst), 1440, 1365, 1075, 1020, $880\left(=\mathrm{CH}_{2}\right.$, out of plane), 780, 740, 730, 690 (out of plane). - MS (96 eV): $m / z=222\left(1 \%, \mathrm{M}^{+}\right), 207(0.5), 168(18), 167(100), 166$ (7), 165 (19), 152 (9), 128 (2), 115 (2).

$$
\mathrm{C}_{17} \mathrm{H}_{18}(222.3) \text { Ber. C } 91.84 \text { H } 8.16 \text { Gef. C } 91.93 \text { H } 7.97
$$

3-Chlor-3-methyl-1-(4-methylphenyl)-1-phenylbutan (farblose Nadeln mit Schmp. $11^{\circ} \mathrm{C}$, aus Petrolether), das analog $3 \mathbf{d}^{\prime}$ aus $\alpha$-(4-Methylphenyl)benzylchlorid $\left(\mathbf{1}^{\prime \prime \prime}\right)$ und Isobuten erhalten wurde, lieferte beim Behandeln mit Kalium-tert-butoxid in der oben beschriebenen Weise $81 \%$ 2-Methyl-4-(4-methylphenyl)-4-phenyl-1-buten (40) als farblose Flüssigkeit mit 
Sdp. $68-78^{\circ} \mathrm{C}(\mathrm{Bad}) / 0.1 \mathrm{~Pa} .-{ }^{1} \mathrm{H}-\mathrm{NMR}\left(\mathrm{CCl}_{4}\right): \delta=1.65\left(\mathrm{~s} ; 3 \mathrm{H}, 2-\mathrm{CH}_{3}\right), 2.23(\mathrm{~s} ; 3 \mathrm{H}, \mathrm{Ar}-$ $\mathrm{CH}_{3}$ ), 2.72 (d, $\left.J=8.1 \mathrm{~Hz} ; 2 \mathrm{H}, 3-\mathrm{H}\right), 4.10$ (t, $\left.J=8.1 \mathrm{~Hz} ; 1 \mathrm{H}, 4-\mathrm{H}\right), 4.60-4.67$ (m; $2 \mathrm{H}$, 1-H), 7.03 und 7.15 (2 s; 9H, Aromaten-H).

$$
\mathrm{C}_{18} \mathrm{H}_{20}(236.4) \text { Ber. C } 91.47 \text { H } 8.53 \text { Gef. C } 91.54 \text { H } 8.40
$$

b) Umsetzung von 2-Methyl-4-(4-methylphenyl)-4-phenyl-1-buten (40) mit Diphenylmethylchlorid (1'): $1.00 \mathrm{~g}(4.23 \mathrm{mmol}) 40$ wurden in $20 \mathrm{ml} \mathrm{CH} \mathrm{Cl}_{2}$ bei $-78^{\circ} \mathrm{C}$ mit einer Lösung von $0.60 \mathrm{~g}$ (4.4 mmol) $\mathrm{ZnCl}_{2}$ in $0.7 \mathrm{ml}$ Ether und $1.4 \mathrm{ml}$ Dichlormethan versetzt. Dazu wurden innerhalb $10 \mathrm{~min} 0.857 \mathrm{~g}(4.23 \mathrm{mmol}) 1^{\prime}$ in $10 \mathrm{ml} \mathrm{CH} \mathrm{Cl}_{2}$ getropf. $\mathrm{Nach} 48 \mathrm{~h}$ wurde auf konz. wäßr. Ammoniak gegossen und das Lösungsmittel abgezogen. Es wurden $1.78 \mathrm{~g}$ einer sehr viskosen Flüssigkeit erhalten, die aus 3-Chlor-3-methyl-1-(4-methylphenyl)-1,5,5triphenylpentan (3n) bestand. $-{ }^{1} \mathrm{H}-\mathrm{NMR}\left(\mathrm{CCl}_{4}\right): \delta=1.08\left(\mathrm{~s} ; 3 \mathrm{H}, 3-\mathrm{CH}_{3}\right), 2.27(\mathrm{~s} ; 3 \mathrm{H}, \mathrm{Ar}-$ $\mathrm{CH}_{3}$ ), 2.50 (d, $\left.J=6 \mathrm{~Hz} ; 4 \mathrm{H}, 2-, 4-\mathrm{H}\right), 4.27$ (br. t, $\left.J=6 \mathrm{~Hz} ; 2 \mathrm{H}, 1-, 5-\mathrm{H}\right), 6.97-7.20$ (m; 19H, Aromaten-H). - MS (70 eV): $m / z=440,438\left(3 \%, 9 \%, \mathbf{M}^{+}\right), 403$ (1), 402 (3), $236(2)$, 235 (2), 221 (3), 181 ( $\left.\mathrm{PhTolCH}^{+}, 100\right), 167\left(\mathrm{Ph}_{2} \mathrm{CH}^{+}, 42\right), 165$ (28).

In gleicher Weise wurde 4n mit $1^{\prime}$ zu 3p und 40 mit $1^{\prime \prime \prime}$ zu 3q umgesetzt: 3-Chlor-3methyl-1,1,5,5-tetraphenylpentan (3p): Farblose meißelförmige Kristalle mit Schmp. $77-79^{\circ} \mathrm{C}$ ( $n$-Pentan/Ether). - ${ }^{1} \mathrm{H}-\mathrm{NMR}\left(\mathrm{CCl}_{4}\right): \delta=1.10\left(\mathrm{~s} ; 3 \mathrm{H}, 3-\mathrm{CH}_{3}\right), 2.52(\mathrm{~d}, J=$ $6.2 \mathrm{~Hz}$; $4 \mathrm{H}, 2-, 4-\mathrm{H}), 4.30$ (t, $J=6.2 \mathrm{~Hz} ; 2 \mathrm{H}, 1-, 5-\mathrm{H}), 7.03-7.25$ (m; 20H, Aromaten-H). MS (70 eV): $m / z=426,424\left(4 \%, 12 \%, M^{+}\right), 389$ (4), 388 (13), 222 (2), 221 (6), 208 (7), 207 (12), 180 (1.5), $167\left(\mathrm{Ph}_{2} \mathrm{CH}^{+}, 100\right)$.

\section{$\mathrm{C}_{30} \mathrm{H}_{29} \mathrm{Cl}$ (425.0) Ber. C 84.78 H 6.88 Gef. C 84.67 H 6.70}

3-Chlor-3-methyl-1,5-bis(4-methylphenyl)-1,5-diphenylpentan (3q): Farbloses Öl. - ${ }^{1} \mathrm{H}-$ NMR $\left(\mathrm{CCl}_{4}\right): \delta=1.08\left(\mathrm{~s} ; 3 \mathrm{H}, 3-\mathrm{CH}_{3}\right), 2.25\left(\mathrm{~s} ; 6 \mathrm{H}, \mathrm{Ar}-\mathrm{CH}_{3}\right), 2.48(\mathrm{~d}, J \approx 6 \mathrm{~Hz} ; 4 \mathrm{H}, 2-$, 4-H), 4.23 (br. t, $J \approx 6 \mathrm{~Hz} ; 2 \mathrm{H}, 1-, 5-\mathrm{H}), 7.00-7.25$ (m; $18 \mathrm{H}$, Aromaten-H). - MS (70 eV): $m / z=454,452\left(0.3 \%, 0.7 \%, \mathbf{M}^{+}\right), 416(0.6), 288(0.3), 274(0.7), 272(2.4), 270(1), 236(13)$, 226 (18), 221 (11), 196 (13), 181 ( $\left.\mathrm{PhTolCH}^{+}, 100\right), 166$ (21), 165 (23).

\section{Konkurrenzexperimente}

In einem 100-ml-Rundkolben wurden etwa $0.40 \mathrm{mmol} 1$ sowie zwei Olefine in solcher Menge eingewogen, daß die Additionsprodukte in relativen Mengen von 0.1 bis 10 entstanden. Es wurde mit $50 \mathrm{ml}$ absol. $\mathrm{CH}_{2} \mathrm{Cl}_{2}$ aufgefüllt, der Kolben auf $-70 \mathrm{bzw}$. $-78^{\circ} \mathrm{C}$ gekühlt und nach Thermostatisierung unter Rühren eine Lösung von $1.90 \mathrm{mmol} \mathrm{ZnCl}_{2}$ in $0.31 \mathrm{ml}$ absol. Ether und $0.62 \mathrm{ml}$ absol. $\mathrm{CH}_{2} \mathrm{Cl}_{2}$ zugesetzt. Nach Beendigung der Umsetzung wurde die Lösung auf ca. $50 \mathrm{ml}$ konz. wäßr. Ammoniak gegossen und durchgeschüttelt. Die organische Phase wurde abgetrennt, über $\mathrm{CaCl}_{2}$ getrocknet, eingeengt und durch HPLC (Tab. 5) analysiert. In einigen Fällen wurde das Diarylmethylchlorid 1 zu einer thermostatisierten Lösung der Olefine und des Katalysators gegeben, was auf die Konkurrenzkonstante keinen Einfluß hatte.

Tab. 6 zeigt die Einwaagen der Reaktanten, die aus Eichkurven ermittelten Produktverhältnisse sowie die nach Gl. (1) resultierenden Konkurrenzkonstanten.

\section{Limonen (4r) und Bis(4-methylphenyl)methylchlorid (1")}

Wie für die anderen Additionen beschrieben, erhielt man durch Umsetzung von $1.90 \mathrm{~g}$ (14.0 mmol) $4 \mathrm{r}$ mit $2.07 \mathrm{~g}(8.97 \mathrm{mmol}) 1^{\prime \prime}$ in Gegenwart von $0.28 \mathrm{~g} \mathrm{ZnCl}_{2}$ und $0.3 \mathrm{ml}$ Ether in $50 \mathrm{ml} \mathrm{CH}_{2} \mathrm{Cl}_{2}$ bei $-78^{\circ} \mathrm{C}$ ein Gemisch der 1:1-Produkte 3ra und 3rb sowie mehrerer stereoisomerer 2:1-Produkte. Durch präparative Mitteldruckchromatographie (LiChroprep 
Si $60,15-25 \mu \mathrm{m}$; Petrolether $\left(40-60^{\circ} \mathrm{C}\right) /$ Ether $(100: 1$, v:v) ließen sich 3ra und 3rb abtrennen. Vermutlich handelt es sich bei 3ra um ein Diastereomerengemisch, während das kristallisierte 3rb sterisch einheitlich sein dürfte.

Tab. 5. Chromatographische Trennungen der bei den Konkurrenzexperimenten anfallenden Produktgemische

\begin{tabular}{|c|c|c|c|c|c|}
\hline Reaktanten & $\begin{array}{l}\text { Stationare } \\
\text { Phase }\end{array}$ & Hobile Phase & & $\begin{array}{l}\text { FluB } \\
\text { (ml/min) }\end{array}$ & $\begin{array}{l}\text { Retentionszeiten } \\
(\min )\end{array}$ \\
\hline $1^{\prime}+4 a / 4 m$ & Nucleosil $5 \mathrm{NO}_{2}$ & \multicolumn{2}{|c|}{ Isooctan/Ether $=99 / 1$} & 1.52 & $8 \mathrm{a}^{\prime}(3.2), 3 \mathrm{a}^{\prime}(5.2), 11^{\prime}(6.1)$ \\
\hline$\underline{1}+4 a / 4 c$ & Nucleosil $5 \mathrm{C}_{1}$. & $\mathrm{CH}_{3} \mathrm{CN} / \mathrm{H}_{2} \mathrm{O}$ & $=82 / 18$ & 1.40 & $3 \mathrm{a}^{\prime}(5.4), 3 \mathrm{c}^{\prime}(5.8), 8 \mathrm{a}^{\prime}(7.3)$ \\
\hline $1 '+4 b / 4 m$ & Nucleosil $5 \mathrm{C}_{1 \mathrm{~s}}$ & $\mathrm{CH}_{3} \mathrm{OH} / \mathrm{H}_{2} \mathrm{O}$ & $=87 / 13$ & 1.00 & $3 \underline{b}^{\prime}(6.5), 8 \mathrm{~b}^{\prime}(8.0), 11^{\prime}(8.6)$ \\
\hline $1^{\prime}+4 c / 4 m$ & Nucleosil $5 \mathrm{C}_{10}$ & $\mathrm{CH}_{3} \mathrm{OH} / \mathrm{H}_{2} \mathrm{O}$ & $=88 / 12$ & 1.21 & $3 c^{\prime}(5.9), 8 b^{\prime}(7.2), 11^{\prime}(7.7)$ \\
\hline$\underline{1}^{\prime}+4 c / 4 f$ & LiChrosorb RP-8 & $\mathrm{CH}_{3} \mathrm{OH} / \mathrm{H}_{2} \mathrm{O}$ & $=85 / 15$ & 1.06 & $3 c^{\prime}(6.1), 8 b^{\prime}(7.1), 3 f^{\prime}(8.3)$ \\
\hline$\underline{1}^{\prime}+4 d / 4 f$ & Nucleosil $5 \mathrm{NO}_{2}$ & \multicolumn{2}{|c|}{ Isooctan/Ether-99/1 } & 1.32 & $3 f^{\prime}(5.9), 3 d^{\prime}(6.4)$ \\
\hline $1^{\prime}+4 d / 4 m$ & Nucleosil $5 \mathrm{NO}_{2}$ & \multicolumn{2}{|c|}{ Isooctan/Ether-99/1 } & 1.52 & $3 \mathrm{~d}^{\prime}(5.3), 11^{\prime}(6.4)$ \\
\hline $1^{\prime}+4 f / 4 m$ & LiChrosorb RP-8 & $\mathrm{CH}_{3} \mathrm{OH} / \mathrm{H}_{2} \mathrm{O}$ & $-85 / 15$ & 1.75 & $11^{\prime}(6.7), 3 f(8.6)$ \\
\hline $1^{\prime}+4 f / 4 g$ & Nucleosil $5 \mathrm{C}_{10}$ & $\mathrm{CH}_{3} \mathrm{OH} / \mathrm{H}_{2} \mathrm{O}$ & $-90 / 10$ & 0.85 & $\begin{array}{l}g^{\prime}(6.4), 3 \mathrm{~g}^{\prime}(7.1), 3 \mathrm{f}^{\prime}(9.0) \\
10 \mathrm{~g}^{\prime}(9.5)\end{array}$ \\
\hline $19+4 f / 4 h$ & Nucleosil $5 \mathrm{NO}_{2}$ & \multicolumn{2}{|c|}{ Isooctan/Ether $=99 / 1$} & 1.40 & $10 h^{\prime}(3.0), 4 f^{\prime}(4.5)$ \\
\hline $11+4 g / 4 h$ & Nuclensil $5 \mathrm{C}_{12}$ & $\mathrm{CH}_{3} \mathrm{OH} / \mathrm{H}_{2} \mathrm{O}$ & $=90 / 10$ & 1.00 & $\begin{array}{l}\underline{g}^{\prime}(6.3), 3 \mathrm{~g}^{\prime}(7.1), 3 \mathrm{~h}^{\prime}(7.9), \\
10 \mathrm{~g}^{\prime}(8.5), 10 \mathrm{~h}^{\prime}(11.8)\end{array}$ \\
\hline $1+4 a / 4 c$ & Nucleosil $5 \mathrm{C}_{1}$. & $\mathrm{CH}, \mathrm{CN} / \mathrm{H}_{2} \mathrm{O}$ & $=82 / 18$ & 1.40 & $\begin{array}{l}3 \mathrm{a}^{\prime \prime}(8.4), 4 \mathrm{c}^{\prime \prime}(9.2), \\
10 \mathrm{a}{ }^{\prime \prime}(11.3), 8 \mathrm{a}{ }^{\prime}(12.3), \\
8 \mathrm{c}^{\prime \prime} \cdot(13.1)\end{array}$ \\
\hline $1 \cdots+4 b / 4 m$ & Nucleosil $5 \mathrm{C}_{10}$ & $\mathrm{CH}_{3} \mathrm{OH} / \mathrm{H}_{2} \mathrm{O}$ & $-98 / 2$ & 1.50 & $3 b^{\prime \prime}(3.9), 11^{\prime \prime}(4.5)$ \\
\hline $1^{\prime \prime}+4 c / 4 m$ & Nucleosil $5 \mathrm{NO}_{2}$ & Isooctan/E & ther $=98 / 2$ & 1.24 & $3 c^{\prime \prime}(5.0), 11^{\prime \prime}(5.6)$ \\
\hline $1^{\prime \prime}+4 e / 4 m$ & Nucleosil $5 \mathrm{C}_{18}$ & $\mathrm{CH}, \mathrm{OH} / \mathrm{H}_{2} \mathrm{O}$ & $-96 / 4$ & 1.50 & $3 \mathrm{e}^{\prime \prime}(5.4), 11^{\prime \prime}(5.8)$ \\
\hline $1^{\prime \prime}+4 f / 4 m$ & Nucleosil $5 \mathrm{NO}_{2}$ & Isooctan/E & ther $=98 / 2$ & 1.54 & $3 f^{\prime \prime}(3.5), 11^{\prime} '(4.1)$ \\
\hline$\underline{1}+4 g / \underline{4 f}$ & Nucleosil $5 \mathrm{C}_{10}$ & $\mathrm{CH}_{3} \mathrm{OH} / \mathrm{H}_{2} \mathrm{O}$ & $=96 / 4$ & 1.50 & $\begin{array}{l}3 g^{\prime} '(4.5), 3 \mathrm{f}^{\prime}+(5.1) \\
10 \mathrm{~g} \cdot 1(5.6)\end{array}$ \\
\hline $1^{\prime \prime}+41 / 4 f$ & Nucleosil $5 \mathrm{C}_{2}$. & $\mathrm{CH}, \mathrm{OH} / \mathrm{H}_{2} \mathrm{O}$ & $=92 / 8$ & 1.50 & $31^{\prime \prime}(7.1), 3 f^{\prime \prime}(7.9)$ \\
\hline$I^{\prime}+4 y^{\prime}\left(\underline{s}^{a}\right)$ & Nucleosil $5 C_{1}$. & $\mathrm{CH}, \mathrm{OH} / \mathrm{H}_{2} \mathrm{O}$ & $=96 / 4$ & 1.50 & $3 s^{\prime} \cdot(4.2), 3 j^{\prime \prime}(5.2)$ \\
\hline $1 \cdots+4 k / 4 m$ & Nucleosil $5 \mathrm{NO}_{2}$ & Isooctan/E & ther $=98 / 2$ & 1.48 & $3 \mathrm{k}^{\prime \prime}(3.7), 11{ }^{\prime \prime}(4.1)$ \\
\hline $1^{\prime \prime}+41 / 4 f$ & Nucleosil $5 \mathrm{NO}_{2}$ & Isooctan/E & ther $=98 / 2$ & 1.25 & $3 \mathrm{f}^{\prime \prime}(4.7), 3 \mathrm{I}^{\prime \prime}(5.3)$ \\
\hline $1^{\prime \prime}+41 / 4 m$ & Nucleosil $5 \mathrm{NO}_{2}$ & Isooctan/E & ther $=98 / 2$ & 1.55 & $31^{\prime \prime}(3.8), 3 \mathrm{~m}^{\prime \prime}(4.1)$ \\
\hline
\end{tabular}

a) $4 \mathrm{~s}=$ Allyltrimethylsilan, das bei der Umsetzung mit 1" 4,4-Bis(4-methylphenyl)-1-buten $\left(3 s^{\prime \prime}\right)$ ergibt ${ }^{16)}$.

4-[1-Chlor-1-methyl-3,3-bis(4-methylphenyl)propyl]-1-methyl-1-cyclohexen (3 ra): Farbloses Öl. - ' $\mathrm{H}-\mathrm{NMR}\left(\mathrm{CCl}_{4}\right): \delta=1.23\left(\mathrm{~s} ; 3 \mathrm{H}, \mathrm{H}_{3} \mathrm{C}-\mathrm{CCl}\right), 1.37-2.23(\mathrm{~m}$; Cyclohexen-4-, 5-, 6-H), 1.60 (br. s; 1- $\mathrm{CH}_{3}$ ), 2.27 (s; 6H, $\mathrm{Ar}-\mathrm{CH}_{3}$ ), 2.40 (d, $J \approx 6 \mathrm{~Hz} ; 2 \mathrm{H}, \mathrm{CH}_{2} \mathrm{CCl}$ ), 4.27 (t, 
$J \approx 6 \mathrm{~Hz} ; 1 \mathrm{H}, \mathrm{CHAr}), 5.27$ (mc; $1 \mathrm{H}$, Vinyl-H), 7.00 (mc; $8 \mathrm{H}$, Aromaten-H). - MS $(70 \mathrm{eV}):$ $m / z=368,366\left(2.5 \%, 7 \%, M^{+}\right), 331(3), 235(11), 208(7), 195(100)$.

Tab. 6. Bestimmung der relativen Alken-Reaktivitäten

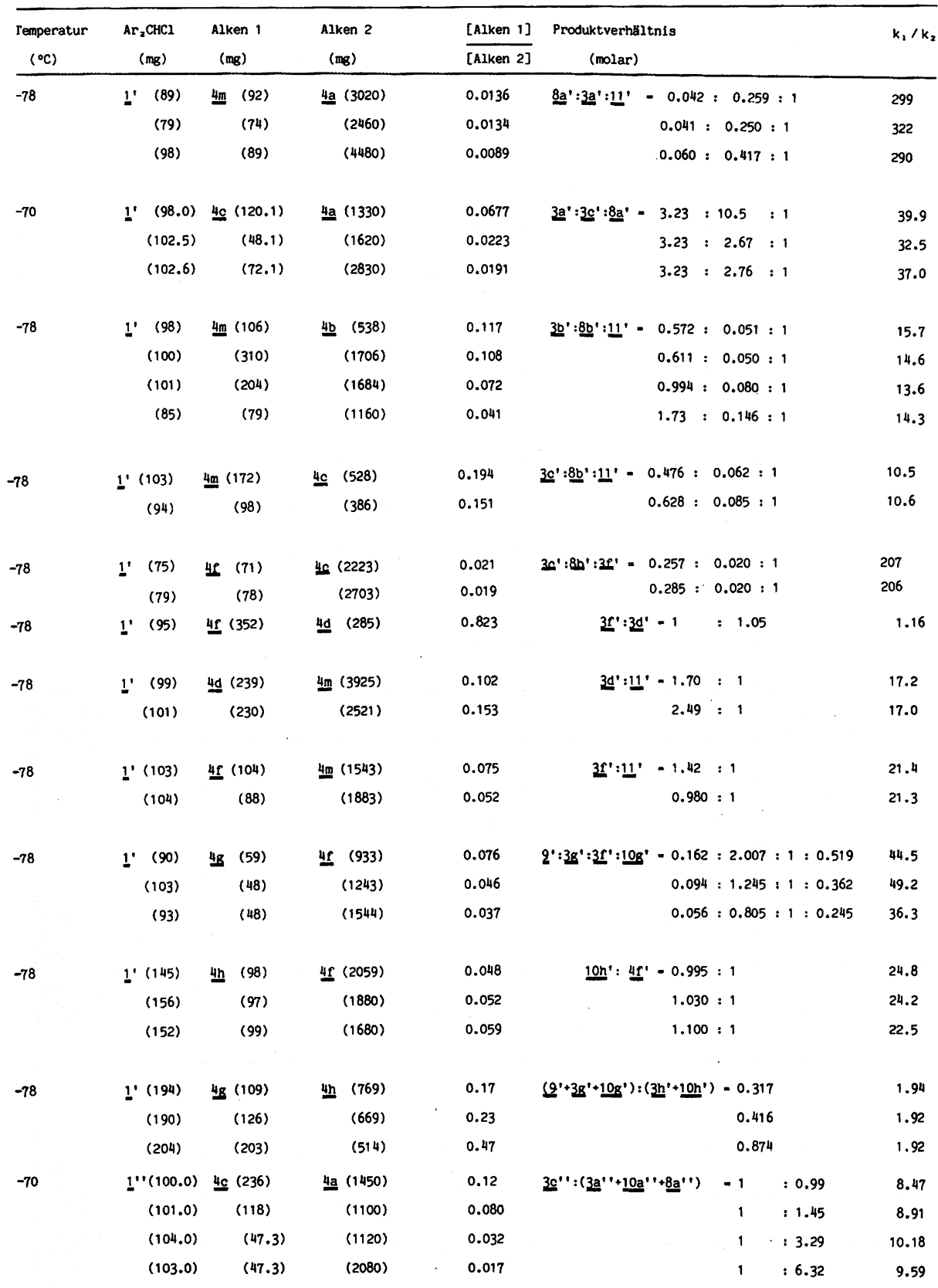

Chem. Ber. 119 (1986) 
Tab. 6 (Fortsetzung)

\begin{tabular}{|c|c|c|c|c|c|c|c|c|}
\hline $\begin{array}{l}\text { Temperatur } \\
\left({ }^{\circ} \mathrm{C}\right) \\
\end{array}$ & $\begin{array}{c}\mathrm{Ar}_{2} \mathrm{CHCl} \\
\text { (mg) }\end{array}$ & $\begin{array}{r}\text { Alk:e: } \\
\text { (mig }\end{array}$ & & $\begin{array}{l}\text { I:ken ? } \\
\text { (ng) }\end{array}$ & $\frac{\text { Alken i] }}{\text { [plken 2] }}$ & $\begin{array}{l}\text { Produktverhältnis } \\
\text { (molar) }\end{array}$ & & $k_{1} / k_{2}$ \\
\hline \multirow[t]{8}{*}{-70} & 1'’(101) & $4 \mathrm{~m}$ & $85:$ & 4b (3119) & $0.018 ?$ & 3b'": 11'" & $=0.264: 1$ & 255 \\
\hline & $(102)$ & & $(106)$ & $(3682)$ & 0.0172 & & $0.280: 1$ & 248 \\
\hline & (113) & & $(74)$ & $(5440)$ & 0.0081 & & $0.715: 1$ & 214 \\
\hline & (120) & & (75! & $(6325)$ & 0.0071 & & $0.871: 1$ & 200 \\
\hline & $(100)$ & & $(84)$ & $(7234)$ & 0.0069 & & $0.824: 1$ & 204 \\
\hline & $(109)$ & & $(85)$ & (7062) & 0.0064 & & $0.981: 1$ & 186 \\
\hline & (102) & & (81) & (9362) & 0.0052 & & $1.180: 1$ & 187 \\
\hline & $(100)$ & & (155) & $(11432)$ & 0.0081 & & $0.784: 1$ & 171 \\
\hline \multirow[t]{3}{*}{-70} & $1 "(100)$ & 4프 & $(81)$ & $4 c \quad(480)$ & 0.101 & $3 c$ '": 11." & $=0.107: 1$ & 124 \\
\hline & $(100)$ & & $(64)$ & $(658)$ & 0.058 & & $0.194: 1$ & 127 \\
\hline & (102) & & (75) & $(1242)$ & 0.036 & & $0.291: 1$ & 125 \\
\hline$\cdot$ & $(105)$ & & $(76)$ & $(1315)$ & 0.034 & & $0.314: 1$ & 121 \\
\hline \multirow[t]{7}{*}{-70} & $1 \cdots(100)$ & 4.j & $(59)$ & 4s (118) & 0.70 & $3 . \mathrm{J}^{\prime \prime}: 3 \mathrm{~s}^{\prime \prime}$ & $-9.33: 1$ & 19.0 \\
\hline & (99) & & $(70)$ & $(197)$ & 0.49 & & $6.96: 1$ & 18.3 \\
\hline & (103) & & (69) & $(305)$ & 0.31 & & $4.51: 1$ & 18.5 \\
\hline & (102) & & $(65)$ & $(601)$ & 0.15 & & $2.20: 1$ & 17.9 \\
\hline & $(100)$ & & $(70)$ & (1201) & 0.081 & & $1.09: 1$ & 15.5 \\
\hline & $(100)$ & & (63) & $(900)$ & 0.097 & & $1.30: 1$ & 15.9 \\
\hline & (101) & & $(66)$ & $(1407)$ & 0.065 & & $0.830: 1$ & 14.5 \\
\hline \multirow[t]{4}{*}{-70} & $1^{\prime \prime}(106)$ & 贸 & $(75)$ & $4 m(1689)$ & 0.043 & 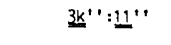 & $-1.83: 1$ & 52.7 \\
\hline & $(107)$ & & $(70)$ & $(2502)$ & 0.027 & & $1.19: 1$ & 53.1 \\
\hline & (107) & & $(67)$ & $(2041)$ & 0.032 & & $1.34: 1$ & 52.3 \\
\hline & (101) & & $(68)$ & $(3224)$ & 0.021 & & $0.908: 1$ & 51.9 \\
\hline \multirow[t]{3}{*}{-70} & $1{ }^{\prime \prime}(104)$ & 4f & (51) & $41 \quad(67)$ & 0.87 & 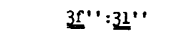 & $: 0.649$ & 2.04 \\
\hline & (102) & & $(175)$ & (71) & 2.81 & & $: 0.186$ & 2.00 \\
\hline & $(104)$ & & (339) & $(68)$ & 5.70 & & $1 \quad: 0.0882$ & 2.05 \\
\hline \multirow[t]{4}{*}{-70} & I' & 4e & (52) & $4 m(2166)$ & 0.032 & 3e' ':11"' & $=0.566: 1$ & 19.7 \\
\hline & $(101)$ & & $(56)$ & $(1803)$ & 0.042 & & $0.730: 1$ & 19.7 \\
\hline & $(104)$ & & (52) & (1336) & 0.052 & & $0.915: 1$ & 20.5 \\
\hline & $(100)$ & & $(73)$ & $(176 ?)$ & 0.056 & & $0.985: 1$ & 19.7 \\
\hline \multirow[t]{4}{*}{-70} & $1^{\prime \prime}(103)$ & 4r & (79) & $4 \ln (1448)$ & 0.061 & $3 \mathbf{f}^{\prime \prime}: 11{ }^{\prime \prime}$ & $=1.470: 1$ & 28.1 \\
\hline & $(101)$ & & $(68)$ & $\left(193^{n}\right)$ & 0.039 & & $0.957: 1$ & 28.1 \\
\hline & $(100)$ & & $(67)$ & $(2431)$ & 0.031 & & $0.741: 1$ & 27.2 \\
\hline & $(110)$ & & $(73)$ & (3068) & 0.027 & & $0.652: 1$ & 27.2 \\
\hline \multirow[t]{4}{*}{-70} & $1{ }^{\prime \prime}(100)$ & 48 & (45) & If $(4008)$ & 0.014 & $38^{\prime \prime}: 3 \mathrm{f}^{\prime \prime} ": 10 \mathrm{~g}^{\prime \prime}$ & $=0.212: 1: 0.096$ & 24.8 \\
\hline & $(100)$ & & (42) & (3013) & 0.017 & & $0.264: 1: 0.121$ & 25.6 \\
\hline & $(105)$ & & $(54)$ & $(2002)$ & 0.032 & & $0.457: 1: 0.221$ & 23.8 \\
\hline & $(105)$ & & $(51)$ & (1365) & 0.045 & & $0.572: 1: 0.282$ & 22.3 \\
\hline
\end{tabular}


Tab. 6 (Fortsetzung)

\begin{tabular}{|c|c|c|c|c|c|c|c|}
\hline $\begin{array}{l}\text { Temperatur } \\
\qquad\left({ }^{\circ} \mathrm{C}\right)\end{array}$ & $\begin{array}{l}\mathrm{Ar}_{2} \mathrm{CHCl} \\
(\mathrm{mg})\end{array}$ & $\begin{array}{l}\text { Alken } 1 \\
\text { (mg) }\end{array}$ & $\begin{array}{c}\text { Alken } 2 \\
\text { (mg) }\end{array}$ & $\frac{[\text { A]ken 1] }}{[\text { Alken 2] }}$ & $\begin{array}{c}\text { Produktverhältnis } \\
\text { (molar) }\end{array}$ & & $k_{1} / k_{2}$ \\
\hline \multirow[t]{4}{*}{-70} & $1{ }^{\prime \prime}(100)$ & 出 (49) & 4f (63) & 0.96 & $3 i^{\prime \prime}: 3 f^{\prime \prime}$ & $-1.600: 1$ & 1.84 \\
\hline & (102) & (52) & (120) & 0.54 & & $0.925: 1$ & 1.86 \\
\hline & (103) & (59) & (182) & 0.40 & & $0.716: 1$ & 1.89 \\
\hline & (106) & (59) & (240) & 0.30 & & $0.534: 1$ & 1.84 \\
\hline \multirow[t]{4}{*}{-70} & $\underline{g} "(104)$ & 41 (76) & 4m (451) & 0.165 & $311^{\prime \prime}: 11$ " & $-1.81: 1$ & 13.4 \\
\hline & $(100)$ & $(77)$ & (815) & 0.093 & & $1.05: 1$ & 13.1 \\
\hline & (111) & $(70)$ & (702) & 0.098 & & $1.11: 1$ & 13.8 \\
\hline & (106) & (68) & (1254) & 0.053 & & $0.652: 1$ & 14.0 \\
\hline
\end{tabular}

2-[Bis(4-methylphenyl)methyl]-1-chlor-1-methyl-4-(1-methylethenyl)cyclohexan (3 rb): Farblose Nadeln mit Schmp. $106-108^{\circ} \mathrm{C}$ (aus Petrolether). - ${ }^{1} \mathrm{H}-\mathrm{NMR}\left(\mathrm{CCl}_{4}\right): \delta=1.18$ (s; $\left.3 \mathrm{H}, 1-\mathrm{CH}_{3}\right), 1.30$ (s; 3H, $\mathrm{H}_{3} \mathrm{C}-\mathrm{C}=$ ), $1.43-2.10$ (m; 7H, 3-, 4-, 5-, 6-H), 2.23 (s; 6H, Ar$\mathrm{CH}_{3}$ ), 3.03 (br. d, $J=10 \mathrm{~Hz} ; 1 \mathrm{H}, 2-\mathrm{H}$ ), 3.87 (d, $J=10 \mathrm{~Hz} ; 1 \mathrm{H}, \mathrm{Ar}_{2} \mathrm{CH}$ ), 4.48 (br. s; $2 \mathrm{H}$, $\left.=\mathrm{CH}_{2}\right), 6.87-7.27\left(\mathrm{~m} ; 8 \mathrm{H}\right.$, Aromaten-H). $-\mathrm{MS}(70 \mathrm{eV}): m / z=368,366\left(3 \%, 8 \%, \mathrm{M}^{+}\right)$, 330 (1.4), 315 (0.3), 230 (0.6), 195 (100).

Zur Bestimmung der intramolekularen Konkurrenzkonstante bei $-70^{\circ} \mathrm{C}$ wurde $1^{\prime \prime}$ in $50 \mathrm{ml} \mathrm{CH} \mathrm{Cl}_{2}$ mit mindestens 10 Äquivalenten $4 \mathrm{r}$ umgesetzt, so daß ausschließlich die 1:1Additionsprodukte 3ra und 3rb gebildet wurden. Die Zusammensetzung wurde durch HPLC-Analyse bestimmt: Nucleosil $5 \mathrm{NO}_{2}$, Isooctan/Ether (98/2), $1.49 \mathrm{ml} / \mathrm{min}$, Retentionszeit $3 \mathrm{ra}=3.6 \mathrm{~min}, 3 \mathrm{rb}=4.0 \mathrm{~min}$.

\begin{tabular}{|c|c|c|c|}
\hline 1" (mmol) & $4 \mathrm{r}(\mathrm{mmol})$ & Katalysator (mmol) & 3ra/3rb \\
\hline 0.433 & 4.29 & $\mathrm{ZnCl}_{2} \cdot 1.57 \mathrm{Et}_{2} \mathrm{O}(2.0)$ & 2.26 \\
\hline 0.442 & 9.34 & (2.0) & 2.42 \\
\hline 0.438 & 4.34 & (2.0) & 2.27 \\
\hline 0.433 & 4.30 & (10) & 2.32 \\
\hline 0.433 & 4.29 & (16) & 2.27 \\
\hline 0.442 & 4.28 & $\mathrm{SnCl}_{4}(0.09)$ & 2.31 \\
\hline 0.442 & 4.31 & (1.8) & 2.85 \\
\hline 0.438 & 4.31 & $(7.2)$ & 3.00 \\
\hline
\end{tabular}

\section{CAS-Registry-Nummern}

1': 90-99-3 / 1": 13389-70-3 / 1"': 779-14-6 / 3a': 36317-61-0 / 3a": 102434-76-4 / $\left(R^{*}, R^{*}\right)-$ $3 b^{\prime}:$ 102434-92-4 / $\left(R^{*}, R^{*}\right)-3 b^{\prime \prime}: 102434-94-6 /\left(R^{*}, S^{*}\right)-3 c^{\prime}: 102434-93-5 /\left(R^{*}, S^{*}\right)-3 c^{\prime \prime}$ :

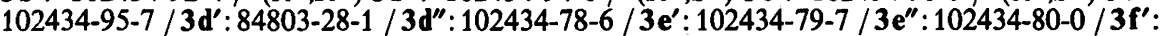
96761-10-3 / 3f": 102434-81-1 / 3g': 102434-82-2 / 3g": 102434-83-3 / 3h': 102434-85-5 / 3i": 102434-86-6 / 3j": 102434-87-7 / 3k": 102434-89-9 / 31": 102492-28-4 / 3n: 102434 98-0 / 3p: 102434-99-1 / 3g: 102436-00-7 / 3ra: 102435-01-8 / 3rb: 102435-02-9 / 3s": 102435-03-0 / 4a: 115-07-1 / 4b: 590-18-1 / 4c: 624-64-6 / 4d: 115-11-7 / 4e: 563-46-2 / 4f: 763-29-1 / 4g: 513-35-9 / 4h: 563-79-1 / 4i: 1120-56-5 / 4j: 1528-30-9 / 4k: 1192-37-6 / 4l: 591-49-1 / 4m: 498-66-8 / 4n: 33925-52-9 / 4o: 102434-97-9 / 4r: 138-86-3 / 4s: 762-72-1 / 8a': 1726-14-3 / 8a": 102434-74-2 / 8b': 66129-95-1 / 8b": 102492-29-5 / 9 g': 42842-40-0 / 9j": 102434-88-8 / cis-10a": 102434-77-5 / trans-10a": 102434-75-3 / 10g': 33508-02-0 / 10g": 102434-84-4 / 10h': 38321-46-9 / 11' (Isomeres 1): 102434-90-2 / 11' (Isomeres 2): 102435-04-1 / 11" (Isomeres 1): 102434-91-3 / 11" (Isomeres 2): 102435-05-2 / PhBr: 10886-1 $/ \mathrm{Ph}_{2} \mathrm{C}=\mathrm{CH}_{2}$ : 530-48-3 $/ \mathrm{PrCO}_{2} \mathrm{Et}$ : 105-54-4 $/ \mathrm{Ph}_{2} \mathrm{CHOH}:$ 91-01-0 / PhCOPh: 11961-9 / (4- $\left.\mathrm{MeC}_{6} \mathrm{H}_{4}\right)_{2} \mathrm{CHOH}: 885-77-8 / 4-\mathrm{MeC}_{6} \mathrm{H}_{4} \mathrm{CH}(\mathrm{Ph}) \mathrm{CH}_{2} \mathrm{CClMe}_{2}$ : 102434-96-8 
1) 1a) P. B. D. de la Mare und R. Bolton, Electrophilic Additions to Unsaturated Systems, Elsevier, Amsterdam 1982. - ${ }^{\text {b) }}$ F. Freeman, Chem. Rev. 75, 439 (1975).

2) 2a) F. Marcuzzi, G. Melloni und G. Modena, J. Org. Chem. 44, 3022 (1979). - ${ }^{2 b)}$ Y. Wang und L. M. Dorfman, Macromolecules 13, 63 (1980).

3) J. P. Kennedy und E. Maréchal, Carbocationic Polymerization, Wiley Interscience, New York 1982.

4) H. Mayr, R. Schneider und R. Pock, Makromol. Chem. 19 (1986), im Druck.

5) Vgl. z. B. H. Mayr, H. Klein und G. Kolberg, Chem. Ber. 117, 2555 (1984).

6) Vorläufige Mitteilung: $H$. Mayr und R. Pock, Tetrahedron Lett. 24, 2155 (1983).

7) H. Mayr, Angew. Chem. 93, 202 (1981); Angew. Chem., Int. Ed. Engl. 20, 184 (1981).

${ }^{8)} H$. Mayr und $W$. Striepe, J. Org. Chem. 48, 1159 (1983).

9) $H$. Mayr und W. Striepe, J. Org. Chem. 50, 2995 (1985).

${ }^{10)}$ R. Pock, H. Mayr, M. Rubow und E. Wilhelm, J. Am. Chem. Soc., zur Publikation eingereicht.

11) R. Pock, H. Klein und H. Mayr, Chem. Ber. 119, 929 (1986).

12) B. Capon und S. P. McManus, Neighboring Group Participation, Vol. 1, S. $58 \mathrm{ff}$., Plenum Press, New York 1976.

13) C. A. Grob, Angew. Chem. 81, 543 (1969); Angew. Chem., Int. Ed. Engl. 8, 535 (1969).

14) C. K. Ingold und F. R. Shaw, J. Chem. Soc. 1949, 575.

15) R. Pock und H. Mayr, Chem. Ber. 119, 2497 (1986), nachstehend.

16) H. Mayr und R. Pock, Tetrahedron, im Druck.

17) B. Giese, Acc. Chem. Res. 17, 438 (1984), und dort zitierte Literatur.

${ }^{18)}$ E. M. Arnett und C. Petro, J. Am. Chem. Soc. 100, 5408 (1978).

19) E. M. Arnett und N. J. Pienta, J. Am. Chem. Soc. 102, 3329 (1980).

20) J. B. Pedley und J. Rylance, Sussex-N.P.L. Computer Analysed Thermochemical Data: Organic and Organometallic Compounds, Sussex 1977.

21) A. Streitwieser jr., Solvolytic Displacement Reactions, S. 95f., McGraw-Hill, New York 1962.

22) 22a) M. T. Bowers, Gas Phase Ion Chemistry, Vol. 2, S. 36, Academic Press, New York 1979. - ${ }^{22 b)}$ R. B. Turner, P. Goebel, B. J. Mallon, W. von E. Doering, J. F. Coburn jr. und M. Pomerantz, J. Am. Chem. Soc. 90, 4315 (1968). - ${ }^{22 c)}$ E. M. Arnett, R. P. Quirk und J. W. Larsen, J. Am. Chem. Soc. 92, 3977 (1970).

23) 23a) G. A. Olah und G. Liang, J. Am. Chem. Soc. 96, 195 (1974). - ${ }^{23 b)}$ G. A. Olah, A. L. Berrier und G. K. Surya Prakash, J. Org. Chem. 47, 3903 (1982).

24) G. Collin, U. Jahnke, G. Just, G. Lorenz, W. Pritzkow, M. Röllig, L. Winguth, P. Dietrich, C.-E. Döring, H. G. Hauthal und A. Wiedenhöft, J. Prakt. Chem. 311, 238 (1969).

${ }^{25)}$ E. V. Couch und J. A. Landgrebe, J. Org. Chem. 40, 1636 (1975).

26) $P$. Asmus und $M$. Klessinger, Tetrahedron 30, 2477 (1974).

${ }^{27)}$ P. Masclet, D. Grosjean, G. Mouvier und J. Dubois, J. Electron Spectrosc. 2, 225 (1973).

28) W. K. Chwang, V. J. Nowlan und T. T. Tidwell, J. Am. Chem. Soc. 99, 7233 (1977).

${ }^{29)}$ R. Huisgen, P. H. J. Ooms, M. Mingin und N. L. Allinger, J. Am. Chem. Soc. 102, 3951 (1980).

30) G. Melloni, G. Modena und U. Tonellato. Acc. Chem. Res. 14, 227 (1981).

${ }^{31)} M . F$. Ruasse, J. E. Dubois und A. Argile, J. Org. Chem. 44, 1173 (1979).

${ }^{32)}$ P. Knittel und T. T. Tidwell, J. Am. Chem. Soc. 99, 3408 (1977).

33) M. L. Poutsma, J. Am. Chem. Soc. 87, 4285 (1965).

34) E. Bienvenue-Goëtz und J.-E. L)ubois, Tetrahedron 34, 2021 (1978).

35) G. H. Schmid und D. G. Garratt in The Chemistry of Double-Bonded Functional Groups (Ed. S. Patai), S. 725f., Wiley, New York 1977.

36) H. J. Bergmann, G. Collin, G. Just, G. Müller-Hagen und W. Pritzkow, J. Prakt. Chem. 314, 285 (1972).

37) A. D. Allen, Y. Chiang, A. J. Kresge und T. T. Tidwell, J. Org. Chem. 47, 775 (1982).

${ }^{38)}$ C. F. H. Allen und S. Converse, Org. Syntheses, Coll. Voll. 1, 226 (1932).

39) Die Zuordnung zu cis- und trans-10a" gelingt aus der 'H-NMR-Absorption von 1-H: $Y$. S. Tsybin, A. V. Kessenikh und R. N. Volkov, Zh. Org. Khim. 5, 806 (1969). 